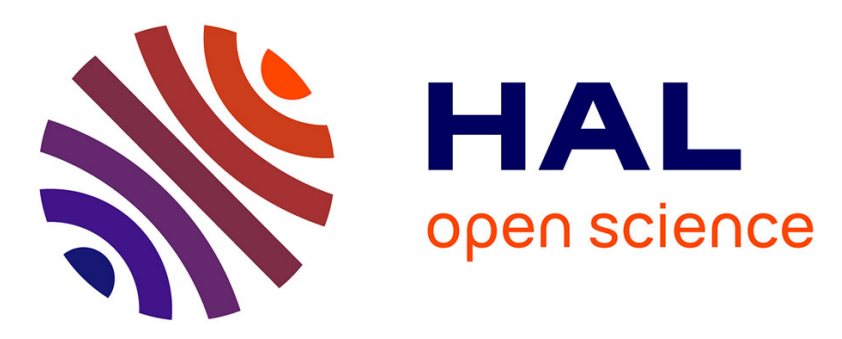

\title{
The non-equilibrium dissipation scaling in large Reynolds number turbulence generated by rectangular fractal grids
}

Shaokai Zheng, P J K Bruce, C Cuvier, J.-M Foucaut, J M R Graham, J C Vassilicos

\section{To cite this version:}

Shaokai Zheng, P J K Bruce, C Cuvier, J.-M Foucaut, J M R Graham, et al.. The non-equilibrium dissipation scaling in large Reynolds number turbulence generated by rectangular fractal grids. Physical Review Fluids, 2021. hal-03217070

\section{HAL Id: hal-03217070 \\ https://hal.science/hal-03217070}

Submitted on 4 May 2021

HAL is a multi-disciplinary open access archive for the deposit and dissemination of scientific research documents, whether they are published or not. The documents may come from teaching and research institutions in France or abroad, or from public or private research centers.
L'archive ouverte pluridisciplinaire HAL, est destinée au dépôt et à la diffusion de documents scientifiques de niveau recherche, publiés ou non, émanant des établissements d'enseignement et de recherche français ou étrangers, des laboratoires publics ou privés. 


\title{
The non-equilibrium dissipation scaling in large Reynolds number turbulence generated by rectangular fractal grids
}

\author{
Shaokai Zheng, ${ }^{1, *}$ P. J. K. Bruce, ${ }^{2}$ C. Cuvier ${ }^{3}$ J.-M. Foucaut,${ }^{3}$ J. M. R. Graham,${ }^{2}$ and J. C. Vassilicos ${ }^{3}, \dagger$ \\ ${ }^{1}$ ARTORG Center for Biomedical Engineering Research, \\ Faculty of Medicine, University of Bern, 3008 Bern, $\mathrm{CH}$ \\ ${ }^{2}$ Department of Aeronautics, Imperial College London, \\ South Kensington Campus, SW7 $2 A Z$ London, UK \\ ${ }^{3}$ Univ. Lille, CNRS, ONERA, Arts et Metiers Institute of Technology, Centrale Lille, \\ UMR 9014 - LMFL - Laboratoire de Mécanique des fluides de Lille - Kampé de Feriet, F-59000 Lille, FR
}

\begin{abstract}
In this study, the turbulence fields generated by a group of modified fractal grid, referred to as the rectangular fractal grids (RFGs), are documented and discussed. The experiments were carried out using hot-wire anemometry in three facilities at Imperial College London, and LMFL in Lille, France. Due to the large Reynolds number of the resulting turbulence, several data processing methods for turbulence properties are carefully evaluated and discussed. Two spectral models were adopted respectively to correct the large and small wavenumber range of the measured spectrum. After the technical discussion, the measurement results are presented in terms of one-point statistics, length scales, homogeneity, isotropy, and dissipation. The main conclusions are twofold. Firstly, the location of maximum turbulence intensity $x_{\text {peak }}$ is shown to be independent of inlet Reynolds number, but dependent on the ratio between the lengths of the largest grid bars in the transverse and vertical directions. This is crucial to the production of prescribed features of turbulent flows in laboratory. Secondly, these RFG-generated turbulent flows are shown to be quasi-homogeneous in the decay region for $x / x_{\text {peak }}>1.5$, but the isotropy is poorer than that of the previous studied fractal square grid-generated turbulence. In the beginning of the decay region, a decreasing pattern of the integral length scale $L_{u}$ and Taylor microscale $\lambda$ was observed for the first time in decaying turbulence, yet the ratio $L_{u} / \lambda$ remained roughly constant along the centerline, so that $C_{\epsilon} \sim R e_{\lambda}^{-1}$, complying with the non-equilibrium scaling relation reported in previous studies for various turbulent flows.
\end{abstract}

\section{INTRODUCTION}

Grid generated turbulence has been studied extensively over the past few decades. In most cases, planar grids with uniform mesh sizes were used to produce flows close to a homogeneous isotropic turbulence. This series of works dates back to Simmons and Salter [1] and Taylor [2], and contributions have been made by many [e.g. 3-9] to study the evolution of various turbulence characteristics.

Over the last decade, research into space-filling fractal square grids, henceforth referred to as FSGs [10] has recorded the existence of a non-equilibrium region where turbulence characteristics (such as various length scales and the dissipation coefficient) evolve differently from the predictions of classical theory. Further, these observations have been confirmed using conventional regular and bi-planar grids $[11,12]$ with measurements in the close vicinity downstream, bringing an alternative perspective to the classical theory of equilibrium turbulence.

The remaining introduction will first summarize the scaling laws of one point statistics derived from grid-generated turbulence (mostly fractal grids but also regular grids), after which the previous discussions on turbulence dissipation rate are briefly reviewed. Here and for the rest of the discussion, $x, y, z$ represent the streamwise, vertical, and transverse directions, respectively, and the origin is always chosen to be the windward geometry center of the grid.

\footnotetext{
* Email: shaokai.zheng@outlook.com

$\dagger$ Email: john-christos.vassilicos@centralelille.fr
} 


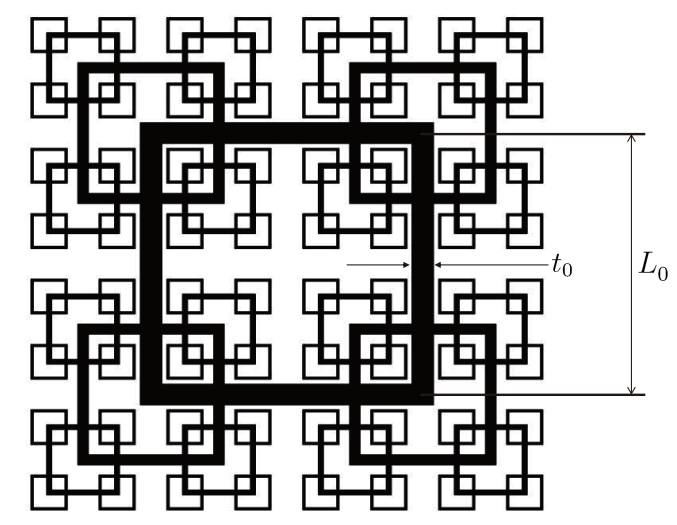

FIG. 1. Example of space-filling square fractal grid, where $L_{0}$ and $t_{0}$ give the length and thickness of the bar at first iteration, respectively.

\section{A. Scaling of turbulence statistics}

The very first study of fractal-generated turbulence was the turbulence generated by three dimensional fractal objects[13], complemented later by simulations of periodic turbulence subjected to fractal/multi-scale forcing[14, 15]. The concept of fractal-generated turbulence was further consolidated by Hurst and Vassilicos [16], where twodimensional grids with three types of fractal-generating patterns were proposed, i.e. cross, I-shaped, and squares.

Out of the three variations of fractal grids, the best homogeneity of decaying turbulence was produced by the space-filling fractal square grids (FSGs), i.e. grids with square patterns (as shown in figure 1 for example). The pattern can be characterized by the fractal iteration number $N_{f}$, the bars' length $L_{j}$ and width $t_{j}$. With relatively low blockage ratios (approximately 25\%), the grid exhibited large turbulence intensities and a protracted production region in comparison to classical-grid generated turbulence with blockage ratios of $44 \%$. The turbulence builds up to a maximum value at a streamwise position $x_{\text {peak }}$, which sets apart the production region $\left(x<x_{\text {peak }}\right)$ and the decay region $\left(x>x_{\text {peak }}\right)$. These authors[16] commented that it might be possible to control $x_{\text {peak }}$ by designing the geometry of the grids. Indeed, it was later shown that both $x_{\text {peak }}$ and the turbulence intensity at the peak can be predicted given the geometry of the grid[17, 18].

The homogeneity and isotropy of such FSG generated turbulence was deemed satisfactory in the decay region[10] around the centerline. Large scale isotropy was least satisfactory. Nevertheless, the ratio of $u^{\prime} / v^{\prime}$ reached values between 1.2 and 1.3, and $L_{u} / L_{v} \approx 2$, where $L_{u}=L_{u u, x}$ and $L_{v}=L_{v v, x}$ are the longitudinal integral length scales of the fluctuating velocities $u$ and $v$, respectively, which was not worse than that of the homogeneous turbulence generated by active grids[8].

Mazellier and Vassilicos [17] performed further experiments on four different FSGs. By relating the wake width $l$ with the bar width $t_{j}\left(j=0, \ldots, N_{f}-1, N_{f}\right.$ being the fractal iteration number $)$ and the streamwise distance $x$ from the bar via $l \sim \sqrt{x t_{j}}[19]$, they introduced the wake interaction length scale $x_{*}=L_{0}^{2} / t_{0}$, where $L_{0}$ is the length of the largest bars and $t_{0}$ is the corresponding largest bar width, both shown in figure 1 . These authors were then able to collapse data from their four grids such that the streamwise location of the peak turbulence intensity was approximately

$$
x_{\text {peak }} \approx 0.45 x_{*}=0.45 \frac{L_{0}^{2}}{t_{0}} .
$$

Valente and Vassilicos [12] confirmed the scaling 1 for various regular grids, with a coefficient which can differ from 0.45 in the range between 0.4 and 0.63. Both mean velocities and turbulence intensities from these four different space-filling FSGs collapsed with such scaling. The flow homogeneity was measured by the ratios $U_{c} / U_{d}$ and $u_{c}^{\prime} / u_{d}^{\prime}$, where $U_{c}$ and $u_{c}^{\prime}$ are the mean velocity and turbulence intensity, respectively, along the centerline of the grid, and $U_{d}$ 
and $u_{d}^{\prime}$ are along the streamwise line behind the corner of the square of second iteration [see 17]. Both of these ratios approached $1 \pm 10 \%$ by $x / x_{*}=0.6$.

Although the scaling relation of equation 1 successfully collapsed the streamwise development of various properties (e.g. Skewness, Flatness, and length scales), it failed when Gomes-Fernandes et al. [18] compared data with different incoming flow conditions. By taking into account the incoming flow turbulence level and the drag coefficient $C_{d}$ of the grid bars, Gomes-Fernandes et al. [18] proposed a length scale

$$
x_{*}^{\prime}=\frac{L_{0}^{2}}{\alpha C_{d} t_{0}}
$$

where $\alpha$ was determined as 0.287 and 0.231 [after 20] for incoming flows with moderate and negligible free-stream turbulence, respectively, and $C_{d}$ was evaluated from the aspect ratio (defined as $A R=d_{0} / t_{0}$, where $t_{0}$ and $d_{0}$ are the width and streamwise thickness of the largest grid bar, respectively), following Bearman and Trueman [21] and Nakamura and Tomonari [22]. After plotting turbulence intensities against $x / x_{*}^{\prime}$, they finally proposed a revised wake interaction length scale

$$
x_{*}^{\text {peak }}=\phi x_{*}^{\prime}=0.21 x_{*}^{\prime}=0.21 \frac{L_{0}^{2}}{\alpha C_{d} t_{0}}
$$

where $\phi=0.21$ was determined empirically so that $x_{*}^{\text {peak }}=x_{\text {peak }}$. Starting from the mean momentum equation of wake flows, the authors also proposed a scaling relation for the centerline turbulence intensity level

$$
\frac{u^{\prime}}{U_{\infty}}=\frac{1}{\beta}\left(\frac{C_{d} t_{0}}{x_{*}^{\text {peak }}}\right)^{1 / 2} g\left(\frac{x}{x_{*}^{\text {peak }}} ; *\right),
$$

where $\beta$ is a constant equal to 2.88 and 2.68 [after 20] for incoming flows with moderate and negligible free-stream turbulence, respectively, and the function $g$ incorporates any residual dependencies that might exist on the boundary or inlet conditions (denoted by $*$ ). The scaled turbulence intensity $\left(u^{\prime} / U_{\infty}\right) \beta\left(C_{d} t_{0} / x_{*}^{\text {peak }}\right)^{-1 / 2}$ collapsed as a function of $x / x_{*}^{\text {peak }}$ for six different inlet conditions as shown by Gomes-Fernandes et al. [18].

\section{B. Turbulence dissipation}

The study of turbulence dissipation has initiated numerous works on different types of turbulent flows over the past few decades. The cornerstone of classical turbulence theory first proposed by Taylor [2] states that $\epsilon=C_{\epsilon} k^{3 / 2} / L_{u}$ (where $\epsilon$ is the dissipation rate, $C_{\epsilon}$ is the dissipation coefficient, $k$ is the turbulent kinetic energy, and $L_{u}$ is the longitudinal integral length scale), with $C_{\epsilon}=$ const, i.e. independent of Reynolds number, for turbulent flows with large local Reynolds numbers $R e_{\lambda} \gg 1$, where $R e_{\lambda}=u^{\prime} \lambda / \nu, u^{\prime}$ is the streamwise fluctuating r.m.s. velocity, $\lambda$ is the Taylor micro scale, and $\nu$ is the kinematic viscosity. This relation is referred to as the equilibrium dissipation scaling, and is usually explained by the Richardson-Kolmogorov equilibrium cascade mechanism [23]. By assuming $\epsilon=15 \nu u^{\prime 2} / \lambda^{2}$, which holds exactly for isotropic turbulence, it follows directly that $L_{u} / \lambda \sim C_{\epsilon} R e_{\lambda}$. This implies that a larger range between $L_{u}$ and $\lambda$ is expected with increasing Reynolds number $R e_{\lambda}$.

On the other hand, the dependence or independence of $C_{\epsilon}$ on boundary/inlet/initial conditions, has been controversial over the decades of turbulence research, with evidence from both experimental and numerical results. Gad-El-Hak and Corrsin [7] showed a dependency of $C_{\epsilon}=\epsilon L_{u} / u^{\prime 3}$ on the initial condition, i.e. the injection rate of their jet grid, and seemed to suggest a smoothly varying $C_{\epsilon}$ with different initial conditions. Sreenivasan [24] summarized several data sets up to that time, and showed that for certain grids $C_{\epsilon}$ approached a constant value for $R e_{\lambda}>100$ when the Reynolds number was varied by varying the inlet flow velocity. These observations were also included in the review by Lumley [25].

More recently, new data and evidence from a number of experimental and numerical works contributed further to the understanding of the behavior of $C_{\epsilon}$ as summarized by Vassilicos [26]. It was not until the work on fractal grids that the dependency of $C_{\epsilon}$ on flow conditions and Reynolds number could be studied more comprehensively and systematically. One outcome has been the discovery of a region with a new non-equilibrium dissipation scaling such 
that the dependency of $C_{\epsilon}$ on Reynolds numbers is given by $C_{\epsilon} \sim R e_{0}^{m} / R e_{L}^{n} \sim R e_{0}^{m / 2} / R e_{\lambda}^{n}$, where $m \approx n \approx 1 ; R e_{0}$ is a global Reynolds number such that $R e_{0}=\left(U_{\infty} L_{0}\right) / \nu$, and $R e_{L}$ is a local Reynolds number given by $R e_{L}=\left(u^{\prime} L_{u}\right) / \nu$. This non-equilibrium dissipation scaling has now been found in various types of turbulent flows [11, 12, 16-18, 27-36].

Later, Valente and Vassilicos [37] studied the non-equilibrium behavior of the dissipation coefficient by comparing measurements behind fractal and regular grids and both confirmed and generalized the finding of Mazellier and Vassilicos [17], showing that $L_{u} / \lambda \propto \sqrt{R e_{0}}$. Their results revealed two distinct regions for $C_{\epsilon}$ such that $C_{\epsilon} \sim R e_{0}^{m} / R e_{L}^{n}$ held for $x_{\text {peak }}<x<5 x_{\text {peak }}$, where $m \approx n \approx 1$, and $C_{\epsilon} \approx$ const for $x>5 x_{\text {peak }}$ with a rather abrupt transition at $x=5 x_{\text {peak }}$. The streamwise extents of these two regions correspond to about the same number of (a few) turnover times[12, 29]. A later study[12] also ruled out the dependency of such a scaling law on the choice of length scales by showing that both longitudinal and transverse length scales follow this non-equilibrium scaling relation, regardless of the inhomogeneity and anisotropy of different flows.

To close this section, it is worth mentioning that previous works on passive grid-generated turbulence (i.e. excluding active grids) were all carried out with regular grids, single square grids and FSGs, or variants of FSGs such as the grid used by Hearst and Lavoie [29]. In all of these grids, all corresponding bars, whether horizontal (in the $z$ direction) or vertical (in the $y$ direction) had the same lengths and thicknesses, i.e. $L_{0}$ and $t_{0}$ are defined irrespective of orientation. In the current study, we introduce and document the turbulence generated by the rectangular fractal grid (referred to as RFG), which is stretched in the transverse $(z)$ direction, and consequently two distinctive lengths and thicknesses are present at each iteration.

\section{Outline}

The current manuscript documents and discusses the turbulence generated by RFGs using experimental data from wind tunnels. In section II, the experimental setup and a detailed description of the RFG are presented first. To rectify the resolution limit of small scale turbulence structures at large Reynolds numbers, some considerations of data processing methods are discribed in section IIC. In the discussion of results, mean statistics of the turbulence are presented first to explore the scaling relations for the wake interaction length scale. The evolution of turbulence length scales is examined in section III C before the homogeneity and isotropy of the flow are tested. The non-equilibrium scaling relation is then studied using the RFG-generated turbulence. Some remarks and suggestions for future work are given in the end to close the manuscript.

\section{EXPERIMENTS}

\section{A. Facilities and experimental setup}

Experiments presented in this study were conducted in three facilities: the Honda wind tunnel and $3 \times 3$ wind tunnel in the Department of Aeronautics at Imperial College London, and the Lille wind tunnel at Lille Fluid Mechanics Laboratory (LMFL). The dimensional details of the test sections at each facility are given in table I. In the Honda and $3 \times 3$ experiments, the pressures before and after the contraction were measured to determine the free stream velocity $U_{\infty}$, whereas in the Lille experiments a Pitot tube placed at the beginning of the test section was used. Further descriptions can be found in [38].

Information about the setup including the range of the traversing systems are listed in table I. For the Honda experiments, single hot-wire measurements were obtained along the centerline of the wind tunnel with spatial resolution $\Delta x=0.4 \mathrm{~m}$ at $y=z=0 \mathrm{~m}$, and along the streamwise direction at $y=330 \mathrm{~mm}, z=0 \mathrm{~m}$, henceforth referred to as center and bar330, respectively. In the $y-z$ plane at $x=2 \mathrm{~m}$, the flow was probed across the span for $-1 \mathrm{~m}<z<1 \mathrm{~m}$ with spanwise resolution $\Delta z=0.1 \mathrm{~m}$ at $y=0.1,0.2,0.3 \mathrm{~m}$, respectively, whereas at $x=8 \mathrm{~m}$, measurements were taken for $0 \mathrm{~m}<z<1 \mathrm{~m}$ with $\Delta z=0.1 \mathrm{~m}$ at $y=0.1,0.2,0.3 \mathrm{~m}$, respectively.

Two point correlation measurements were also taken in the Honda wind tunnel to study the integral length scales in the $y$ direction. The fixed wire was placed on the center plane at two locations $(x, z)=(6 \mathrm{~m}, 0 \mathrm{~m})$, and $(x, z)=$ $(8 \mathrm{~m}, 0 \mathrm{~m})$, respectively, with the other wire moving up to a separation of $360 \mathrm{~mm}$ to measure the correlation functions $R_{u u, y}$. 
TABLE I. Summary of wind tunnel and measurement specifications in different experiments. The $x, y, z$ represents the streamwise, vertical, and transverse directions, respectively. Packets of data defines the number of independent pieces of data samples acquired. The low-pass filter is applied within the anemometer before the data is recorded.

\begin{tabular}{rccc}
\hline \hline Facility Name & Honda & $3 \times 3$ & Lille \\
Contraction Ratio & $9: 1$ & $9: 1$ & $5.4: 1$ \\
Cross Section Area & $3 \mathrm{~m} \times 1.5 \mathrm{~m}$ & $0.9 \mathrm{~m} \times 0.9 \mathrm{~m}$ & $2 \mathrm{~m} \times 1 \mathrm{~m}$ \\
Test Section Length & $8 \mathrm{~m}$ & $4.2 \mathrm{~m}$ & $21 \mathrm{~m}$ \\
$x$ Traverse Range & {$[2 \mathrm{~m}, 8 \mathrm{~m}]$} & {$[0.8 \mathrm{~m}, 4.1 \mathrm{~m}]$} & {$[0.9 \mathrm{~m}, 18.6 \mathrm{~m}]$} \\
$y$ Traverse Range & {$[0 \mathrm{~m}, 0.36 \mathrm{~m}]$} & {$[-0.15 \mathrm{~m}, 0.15 \mathrm{~m}]$} & {$[-0.327 \mathrm{~m}, 0 \mathrm{~m}]$} \\
$z$ Traverse Range & {$[-1 \mathrm{~m}, 1 \mathrm{~m}]$} & -- & -- \\
Freestream Velocities $U_{\infty}$ & $5,10,15,17 \mathrm{~m} / \mathrm{s}$ & $6,10 \mathrm{~m} / \mathrm{s}$ & $6,9 \mathrm{~m} / \mathrm{s}$ \\
Freestream Turbulence & $0.2 \%$ & $0.1 \%$ & $0.2 \%$ \\
Hot-Wire Anemometer DANTEC Streamline DANTEC Streamline & TSI IFA 300 \\
Hot-Wire Type & Single Wire & Cross Wire & Single Wire \\
Hot-Wire Diameter & $5 \mathrm{micron}$ & $5 \mathrm{micron}$ & $5 \mathrm{micron}$ \\
Hot-Wire Length & $1 \mathrm{~mm}$ & $1 \mathrm{~mm}$ & $1.25 \mathrm{~mm}$ \\
Sampling Frequency & $100 \mathrm{kHz}$ & $125 \mathrm{kHz}$ & $50 \mathrm{kHz}$ \\
Sampling Time & $200 \mathrm{~s}$ & $300 \mathrm{~s}$ & $600 \mathrm{~s}$ \\
Packets of Data & 1 & 2 & 3 \\
Low-Pass Filter & $30 \mathrm{kHz}$ & $30 \mathrm{kHz}$ & $20 \mathrm{kHz}$ \\
& & & \\
\hline \hline
\end{tabular}

In the $3 \times 3$ wind tunnel experiments, two streamwise profiles were taken using a cross wire along the centerline $(y=z=0 \mathrm{~m})$ and behind the center of the largest horizontal bar $(y=-0.144 \mathrm{~m} z=0 \mathrm{~m})$ with a streamwise step of $\Delta x=0.3 \mathrm{~m}$, referred to as center and bar, respectively. Profiles were obtained at $U_{\infty}=6 \mathrm{~m} / \mathrm{s}$ and $U_{\infty}=10 \mathrm{~m} / \mathrm{s}$ to vary the inlet Reynolds number, and two packets of $300 \mathrm{~s}$ samples were acquired at each data point.

The cross hot-wire was calibrated at the beginning and end of every data set acquired using the look-up table method proposed by Lueptow et al. [39] for five velocities from $3 \mathrm{~m} / \mathrm{s}$ to $19 \mathrm{~m} / \mathrm{s}$ and seven angles ranging from $-27^{\circ}$ to $27^{\circ}$. The cross wire was mounted on a servo motor and was aligned with the $x-y$ plane. The temperature and free stream velocity were monitored during all acquisitions, and the data was discarded if temperature variation $\Delta T_{a}=T_{a, \max }-T_{a, \min }$ was larger than $0.1^{\circ} \mathrm{C}$, or the inlet velocity $U_{\infty}$ varied by more than $\pm 1.5 \%$ of the target value.

In the Lille experiments, two profiles were measured from $x=0.86 \mathrm{~m}$ to $18.6 \mathrm{~m}$ along the centerline and behind the horizontal bar $(y=-0.327 \mathrm{~m}, z=0 \mathrm{~m})$, referred to as center, and bar, respectively. Two free stream velocities were used, i.e. $U_{\infty}=6 \mathrm{~m} / \mathrm{s}$ and $9 \mathrm{~m} / \mathrm{s}$.

During the acquisition in the Lille experiment, the incoming velocity was monitored continuously and was found to fluctuate by less than $\pm 0.5 \% U_{\infty}$. The wind tunnel is also equipped with a temperature regulator. The fluctuation of ambient temperature was monitored at all times to be within $\pm 0.15^{\circ} \mathrm{C}$ of the calibration temperature at $17^{\circ} \mathrm{C}$. The data acquisition would restart if either the velocity or temperature variations exceeded these limits, and three packets of $600 \mathrm{~s}$ samples were acquired at each location.

Note that the Lille wind tunnel has a $93 \mathrm{~mm}$ long grit 40 sandpaper (mean roughness 425 micron) attached to the bottom and the top of the tunnel walls upstream of the test section. This feature was not removable, and the turbulence far downstream is inevitably affected by the growing boundary layer, especially for measurements behind the horizontal bar, which was only $0.17 \mathrm{~m}$ above the bottom wall. However, the thickness of the tunnel's boundary layer was found to be $0.17 \mathrm{~m}$ at $x=12 \mathrm{~m}$ downstream of the grid, which is far into the decay region of the turbulence.

\section{B. Grid design}

The original motivation to design a rectangular fractal grid, or RFG, was threefold: (i) stretch the size in $z$ and therefore increase the integral length scale, (ii) test the interaction length scale $x_{*}^{\text {peak }}$ previously proposed for FSGs, 


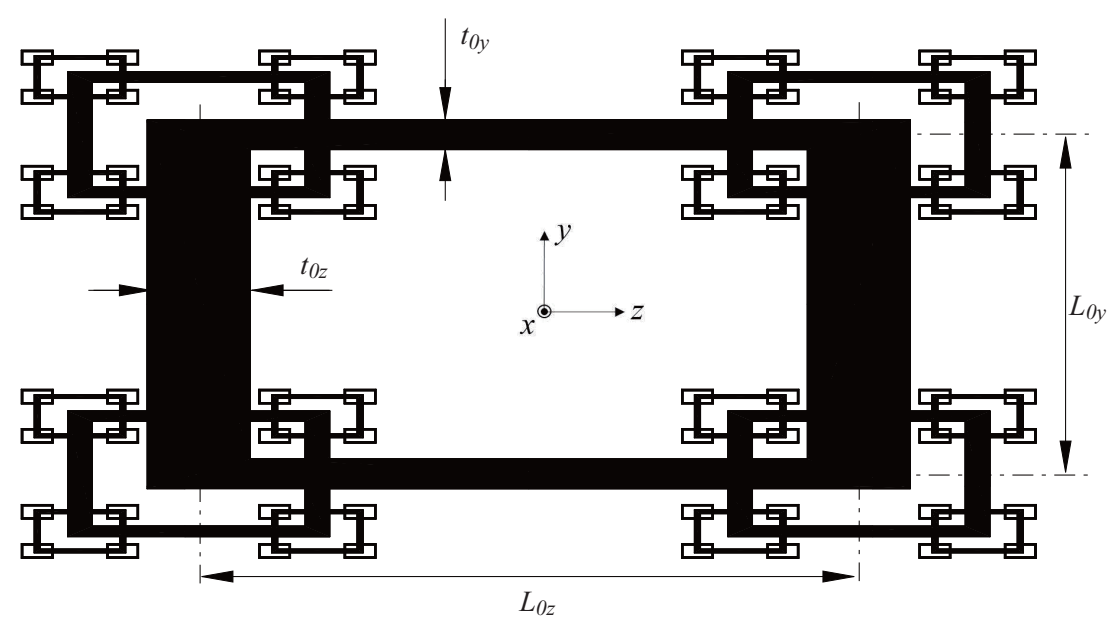

FIG. 2. Rectangular fractal grid for the Honda and Lille wind tunnel. Dimensions mark the first iteration of the grid.

TABLE II. Dimensions of the rectangular fractal grid, where $L, t, d$ correspond to length, width and depth of the bar, respectively, and $j=0,1, \ldots N_{f}-1$, where $N_{f}=4$ is the number of fractal iterations. The subscript $y$ and $z$ gives the direction of the dimension, e.g. $L_{0 y}$ means the length of the bars of the first iteration in the $y$ direction.

\begin{tabular}{ccccc}
\hline \hline$j$ & 0 & 1 & 2 & 3 \\
$L_{j z}(\mathrm{~mm})$ & 1954 & 703 & 253 & 91 \\
$L_{j y}(\mathrm{~mm})$ & 1004 & 341 & 116 & 39 \\
$t_{j z}(\mathrm{~mm})$ & 305 & 73 & 18 & 4 \\
$t_{j y}(\mathrm{~mm})$ & 88 & 31 & 11 & 4 \\
$d_{j z}(\mathrm{~mm})$ & 184 & 45 & 15 & 15 \\
$d_{j y}(\mathrm{~mm})$ & 54 & 19 & 15 & 15 \\
& & & & \\
\hline
\end{tabular}

and (iii) examine the non-equilibrium dissipation scaling [26] when two large length scales of the grid are present.

The first RFG was designed to fit in the Honda wind tunnel (see figure 2). Detailed dimensions of this grid are given in table II. In addition, the grid was designed with a third dimension $d$, which is the depth of the bar in the streamwise direction. This dimension was chosen to give each bar an aspect ratio AR (equal to the streamwise thickness $d$ over the transverse width $t$ ) of 0.61 where possible so that the drag coefficient $C_{d}$ of each bar could be maximized [21]. The last two iterations of the grid, however, have larger aspect ratios of approximately $A R=1$ and $A R=3.75$, respectively, due to durability concerns during manufacturing.

The grid was manufactured from CNC routed plywood sheets, and the depth was modified using foam blocks attached to the downstream side of the grid bars. The grid was then mounted in the wind tunnel with two supporting aluminum profiles attached to the downstream side of the two largest vertical bars. A smaller version of the grid (scaled down by a ratio of 1.54) was manufactured to fit in the Lille wind tunnel with same material and specifications, except for the smallest grids. Due to the glass surface of the Lille wind tunnel, it was mounted on two horizontal aluminum profiles with rubber pads at each end to hold on the side walls, attached to the largest horizontal bars of the grid.

For the $3 \times 3$ experiments, the grid was scaled down by a ratio of 3.5 , and two mirrored halves of it were added at both ends in the vertical direction to fit into the square test section, as shown in figure 3 . The grid was made of laser cut acrylic sheets and was fitted on a wooden frame to support the largest bars. As with the other grids, the bar thickness were adjusted to achieve $A R=0.61$ using foam blocks attached to the grid bars.

The dimensions of the largest bars in each experiment are described in table III. Note that since there are two large 


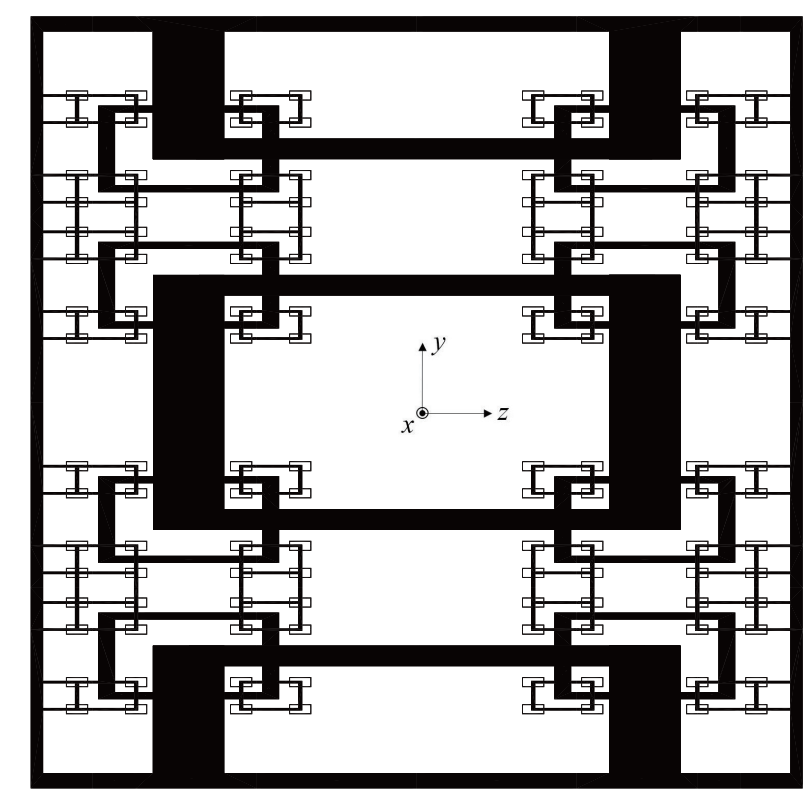

FIG. 3. Rectangular fractal grid for the $3 \times 3$ wind tunnel with frame.

TABLE III. Largest bar size, wake interaction length scales, and the total blockage ratios of the rectangular fractal grids in all experiments, where $x_{y *}^{\text {peak }}=0.21\left(L_{0 y}^{2}\right) /\left(\alpha C_{d} t_{0 y}\right)$ and $x_{z *}^{\text {peak }}=0.21\left(L_{0 z}^{2}\right) /\left(\alpha C_{d} t_{0 z}\right)$. The actual streamwise location for maximum turbulence intensity $x_{\text {peak }}(\mathrm{m})$ is also given, which is further elaborated in section III B.

\begin{tabular}{lcccccccc}
\hline \hline \multicolumn{1}{c}{$L_{0 z}(\mathrm{~m})$} & $t_{0 z}(\mathrm{~m})$ & $x_{z *}^{\text {peak }}(\mathrm{m})$ & $L_{0 y}(\mathrm{~m})$ & $t_{0 y}(\mathrm{~m})$ & $x_{y *}^{\text {peak }}(\mathrm{m})$ & $\sigma(\%)$ & $x_{\text {peak }}(\mathrm{m})$ \\
Honda & 1.954 & 0.308 & 3.924 & 1.004 & 0.088 & 3.591 & 33.4 & 5.20 \\
Lille & 1.272 & 0.199 & 2.557 & 0.654 & 0.057 & 2.339 & 34.3 & 3.79 \\
$3 \times 3$ & 0.558 & 0.087 & 1.123 & 0.287 & 0.025 & 1.032 & 33.8 & 1.72 \\
& & & & & & & \\
\hline \hline
\end{tabular}

scales, there are also two wake interaction length scales $x_{y^{*}}^{\text {peak }}=0.21\left(L_{0 y}^{2}\right) /\left(\alpha C_{d} t_{0 y}\right)$ and $x_{z *}^{\text {peak }}=0.21\left(L_{0 z}^{2}\right) /\left(\alpha C_{d} t_{0 z}\right)$, calculated using the two corresponding dimensions marked by subscripts $y$ and $z$. Note that $x_{y *}^{p e a k} \neq x_{z *}^{p e a k}$ due to the difference of grid bar dimensions in the $y$ and $z$ directions. The blockage ratios for all these grids are very similar at around $33.8 \%$, taking into account the mounting/supporting elements, which is only slightly larger than $\sigma=25 \%$ to $32 \%$ for the square fractal grids used by Valente and Vassilicos [27].

\section{Data processing}

Due to the lack of thermal control in the Honda wind tunnel, temperature variations between different runs as large as $5{ }^{\circ} \mathrm{C}$ were measured during a day of measurement, so a temperature correction method was implemented for the data taken in the Honda experiment. The method is based on the relation that $E_{0}^{2}=A^{\prime}+B^{\prime} T_{a}[38,40]$ at any given free stream velocity, where $E_{0}$ is the output voltage from the anemometer, $A^{\prime}$ and $B^{\prime}$ are fitting constants, and $T_{a}$ is the ambient temperature. The corrected calibration curves taken at different ambient temperatures were successfully collapsed (see figure 4), and the same procedure was employed for all hot-wire data from the Honda experiments before further analysis.

Another issue frequently encountered in experimental works is that the largest scales of the flow are often insuf- 


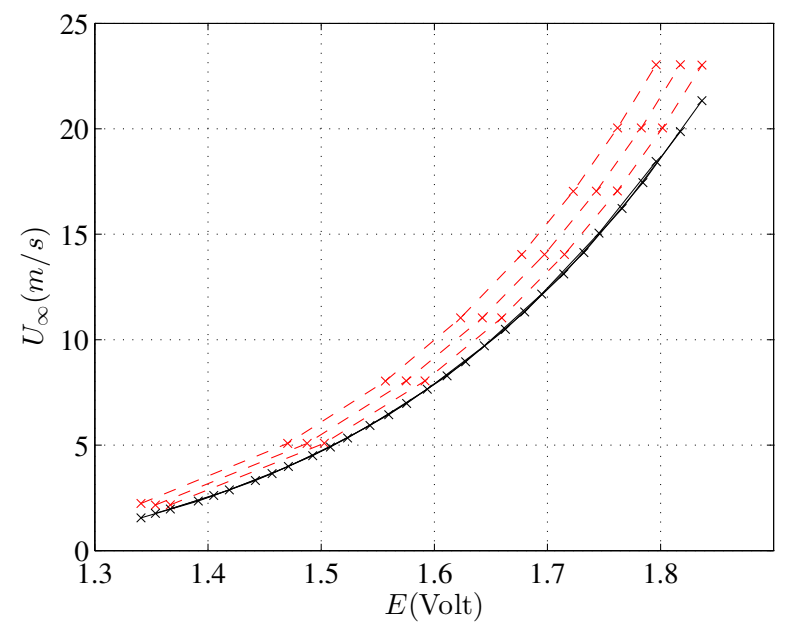

FIG. 4. Original calibration curves at different temperatures (red), and corrected curves (black).

ficiently resolved due to limited sample size. In order to calculate the longitudinal integral length scale $L_{u}$, several methods were compared. The method of integrating auto-correlation functions is not used here due to the ambiguity in the choice of integration limit [41]. These results are also affected by the piecewise sample size if one takes the average of integral length scales calculated from shorter pieces of the samples (see appendix A). Based on the WienerKhinchin theorem, the auto-correlation function and the power spectral density is a Fourier pair, and therefore the power spectral density provides another option to calculate $L_{u}$. In practice, the inertial range of the power spectrum density is first fitted to a model to extrapolate the low wavenumber range of the spectrum, and the integral length scale is calculated using $L_{u}=\pi E_{11}(k=0) /\left(2 u^{\prime 2}\right)$, where $k$ is the wavenumber defined as $k=2 \pi f / U$ ( $f$ is the frequency in time), and $u^{\prime}$ is the streamwise turbulence r.m.s. velocity. To calculate wavenumber $k$, the spatial signal is converted from the time series using Taylor's hypothesis, where the local mean velocity $U$ is taken as the convection velocity. To justify the use of Taylor's hypothesis, some preliminary comparison with PIV (Particle Image Velocimetry) data captured around $x_{\text {peak }}$ was examined (see figure 2.7 of [38]), and it was shown that the spectra produced from both measurement techniques agreed well over the inertial sub-range and the beginning of dissipation range.

To model the spectrum, one of the most frequently used is the von Kármán model [42], which can be written in a one-dimensional form as

$$
E_{11}^{+}(k)=\frac{2 u^{\prime 2} L_{u u, x}}{\pi} \frac{1}{\left\{1+\left[k L_{u u, x} B(1 / 2,1 / 3) / \pi\right]^{2}\right\}^{5 / 6}}
$$

where $k$ is the wave number, and $B$ is the beta function related to the $\Gamma$ function by $B(1 / 2,1 / 3)=\Gamma(1 / 2) \Gamma(1 / 3) / \Gamma(5 / 6)$. More recently, another spectral model was proposed by Wilson [43] based on Meijer's $G$-function (referred to as the $G$-model in this text), of which the one dimensional form can be written as

$$
E_{11}^{*}(k)=\frac{2 u^{\prime 2} l}{3 B(1 / 2,1 / 3+b)}\left(k^{2} l^{2}\right)^{-5 / 6} B_{k^{2} l^{2} /\left(1+k^{2} l^{2}\right)}(5 / 6, b),
$$

where $B_{x}$ is the incomplete beta function. This model includes two fitting variables $l$ and $b$, and is therefore more accurate than the von Kármán model [43] in terms of adjusting the energy level for the inertial sub-range and energycontaining range independently.

Figure 5 shows an example to compare these two models with experimental data, fitted using nonlinear regression in the least squares sense. It is clear that the von Kármán model underestimates the spectral levels in the energycontaining (low wavenumber) range, as reported by Wilson [43]. The sum of squared residuals for the $G$-model is also smaller. Therefore the $G$-model is used henceforth in this study to correct the spectra in the energy-containing range and to calculate integral length scales in the following discussion. Note that the $G$-model might introduce unwanted 


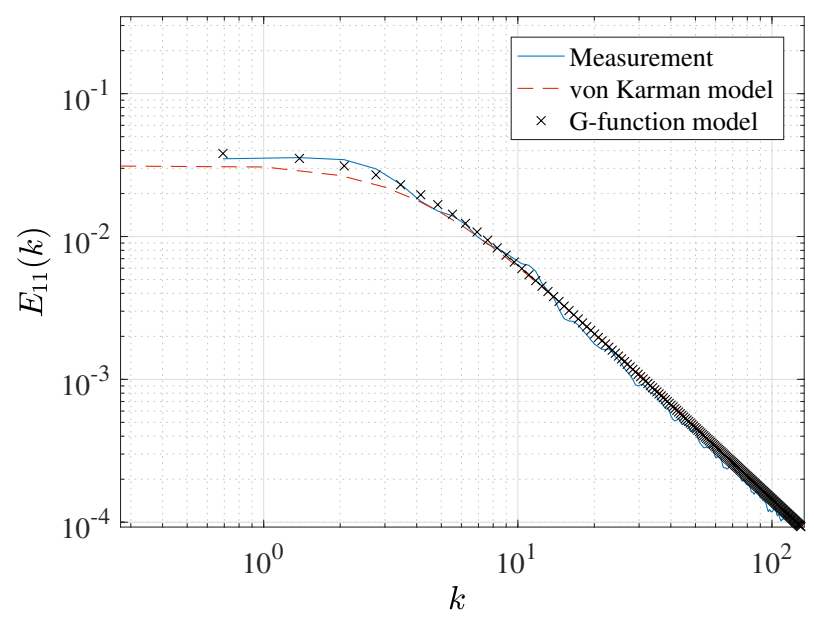

FIG. 5. Comparison of two spectra models against experimental data taken from Honda wind tunnel, in the decay region of RFG generated turbulence.

error if the Reynolds number is small, such that the inertial sub-range does not show a definite $-5 / 3$ slope. In that case, further care must be taken to choose an appropriate spectrum model.

While the inertial range of spectra can be corrected by the $G$-model method, the dissipation range of spectra is also of concern. This part of a spectrum usually suffers from electrical noise which shows up as spurious rises at the end of the spectrum proportional to $k^{2}[44,45]$. This rise can lead to overestimation of the dissipation rate $\epsilon$ because of the $k^{2}$ term in the integrand. Furthermore, at sufficiently large Reynolds number, the Kolmogorov length scale sometimes cannot be resolved due to limited resolution of the hot wire, so it is often desirable to extrapolate the spectra to obtain higher wavenumber components. Although there have been many discussions on spectral models for the large wavenumber range [46, 47], we refer to the method discussed by Pearson [48] and Zhou et al. [49], such that for sufficiently large $k$, the spectrum $E_{11}(k)$ has the form $k^{-5 / 3} \exp (\alpha k+\beta)$, where $\alpha$ and $\beta$ are constants. Thus a linear extrapolation can be applied to $\operatorname{In}\left[k^{5 / 3} E_{11}(k)\right]$ with respect to $k$. Antonia [45] improved the form to $E_{11}(k) \sim k^{m} \exp (\alpha k+\beta)$, where the exponent $-5 / 3$ was substituted by the variable $m$ as he found that the magnitude of $m$ increases with $R e_{\lambda}$. The actual value of $m$ (approximately of 2 ) is chosen by trial and error to give the best fit. The dependence of this method on the choice of fitting wavenumber range was found to be negligible. Extrapolations performed based on ranges from $2 \mathrm{kHz}$ to $5 \mathrm{kHz}$ and $3 \mathrm{kHz}$ to $6 \mathrm{kHz}$, respectively, give $1.8 \%$ difference in the dissipation rate $\epsilon$ calculated by integrating the corrected spectrum. One example of the corrected result is given in figure 6 . It can be observed that the re-sampled spectrum correctly represents the original one in the dissipation range.

For calculations using fluctuating velocity signals, the signals are filtered first. The cutoff frequency is chosen to be $f_{c}=2.3 f_{\text {noise }}$, where $f_{\text {noise }}$ is the frequency where $f^{2} E(f)$ has a minimum in the high frequency range. The constant 2.3 compensates for the Nyquist limit, and the transition band (the region between pass band and stop band) of the filter.

Note that the iterative filtering method adopted in $[29,50]$ was also tested. This method uses the Kolmogorov frequency $f_{\eta}$ as a reference to check the selection of the cutoff frequency $f_{c}$, and recursively reduces $f_{c}$ until the small scale properties such as $\eta$ and $f_{\eta}$ converge. The results, however, depend theoretically on the parameters of the filter, because the filtered spectrum is always attenuated by the transfer function of the digital filter itself, and the converged results do not necessarily represent the true values of small scale properties. For these reasons, the aforementioned extrapolation method is used to correct for the high frequency range of signals. 


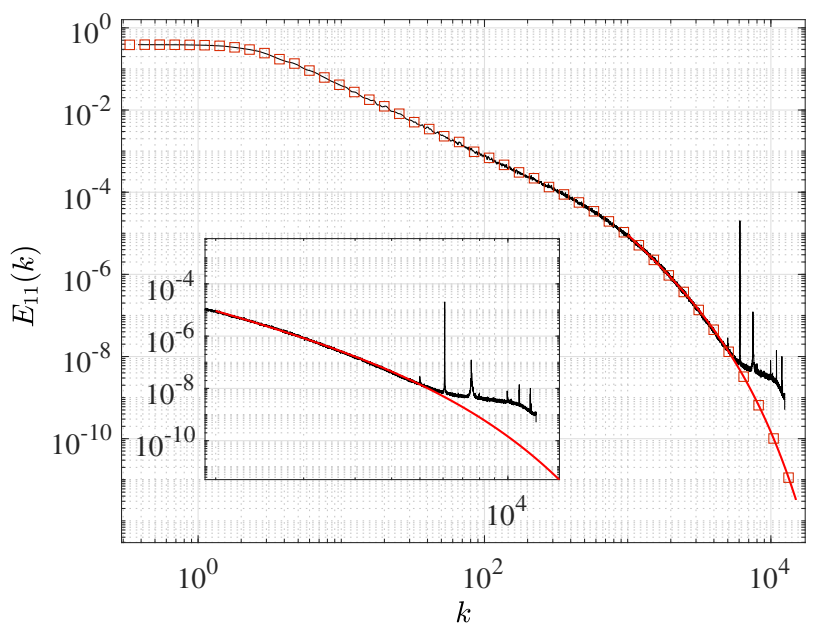

FIG. 6. Re-sampled spectra (squares) after the extrapolation (red lines) from the Honda centerline data at $x / x_{p e a k}=1.08$, $U_{\infty}=10 \mathrm{~m} / \mathrm{s}$.

\section{Experimental uncertainties}

Finally, the results of different calculation methods at selected locations are summarized in table IV to give an overview of the uncertainties. For turbulence intensities calculated using the fluctuating velocity signals and corrected spectra the differences are all well within $\pm 1 \% U_{\infty}$. The longitudinal integral length scales are computed by $L_{u}^{+}=$ $\pi E_{11}^{+}(0) /\left(2 u^{\prime 2}\right)$ (using the spectrum $E_{11}^{+}(k)$ corrected by the von Kármán spectrum model), and $L_{u}^{*}=\pi E_{11}^{*}(0) /\left(2 u^{\prime 2}\right)$ (using the spectrum $E_{11}^{*}(k)$ corrected by the $G$-function model), respectively. Their differences are all within $\pm 5 \%$ from the mean value of $L_{u}^{+}$and $L_{u}^{*}$. However, there is always an underestimation by the von Kármán spectrum model, which is in agreement with the discussions found in Wilson [43].

In terms of the Taylor microscale $\lambda=u^{\prime} /\left(\overline{(d u / d x)^{2}}\right)^{1 / 2}$, they are compared between $\tilde{\lambda}$ (where the velocity gradient is calculated from the filtered fluctuating velocity) and $\lambda^{*}$ (where the velocity gradient is calculated by integrating the G-model corrected spectrum). The difference between $\tilde{\lambda}$ and $\lambda^{*}$ at $x / x_{\text {peak }}=1$ is $\pm 4 \%, \pm 10 \%$ and $\pm 3 \%$ for the Honda, Lille and $3 \times 3$ experiment, respectively. The large error in the Lille experiment can be attributed to the lower sampling/filter frequency due to hardware limitation, as presented in table I. At such large Raynolds numbers, the high frequency components of velocity fluctuations tend to be under-resolved, leading to underestimated velocity gradients and consequently an overestimation of the Taylor microscale. Nevertheless, this defect is assumed to be rectified by the spectrum extrapolation method introduced above. The difference between $\tilde{\lambda}$ and $\lambda^{*}$ in the Lille experiment decreases with Reynolds number down to $\pm 3 \%$ at $x / x_{\text {peak }}=2.73$.

To estimate the dissipation rate, we first calculate both $\tilde{\epsilon}_{i s o}=15 \nu \overline{(d u / d x)^{2}}$, where $\overline{(d u / d x)^{2}}$ is computed from the filtered fluctuating velocity signal, and $\epsilon_{i s o}^{*}=15 \nu \int k^{2} E_{11}^{*}(k) d k$ using the isotropic assumption, where $E_{11}^{*}(k)$ is the G-function corrected spectra. It is noticed that $\epsilon_{i s o}^{*}$ is always larger than $\tilde{\epsilon}_{i s o}$ (table IV), especially at large Reynolds numbers. This is explained by the filtering of the signal and the spectra extrapolation that compensates for the large wavenumber range. For the Honda and $3 \times 3$ experiments, the differences between $\tilde{\epsilon}_{\text {iso }}$ and $\epsilon_{i s o}^{*}$ are $\pm 4 \%$ and $\pm 7 \%$ from their mean values, respectively, at $x / x_{\text {peak }}=1$, where turbulence intensity is largest.

For the Lille experiment, however, the discrepancy between $\tilde{\epsilon}_{i s o}$ and $\epsilon_{i s o}^{*}$ is $\pm 19 \%$ at $x / x_{\text {peak }}=1$, and decreases to $\pm 6 \%$ at $x / x_{\text {peak }}=2.73$. This is consistent with the observations of Taylor microscale, such that the high frequency velocity fluctuations are under-resolved. The corrected spectra are therefore used to estimate dissipation rates.

For full disclosure, the iterative filtering method proposed by Mi et al. [50] was tested too and did give a smaller value of $\epsilon_{\text {iso }}=6.8 \mathrm{~m}^{2} \mathrm{~s}^{-3}$ at $x / x_{\text {peak }}=1$ in the Lille experiment. While it does seem to reduce the discrepancy, the method suffers from the attenuation of the digital filter and the ambiguity of converged length scale (as discussed in section II C), and therefore does not suffice as a better choice. 
TABLE IV. Comparison of turbulence characteristics in different experiments along the centerline calculated using different methods. The integral length scale are calculated as $L_{u}^{+}=\pi E_{11}^{+}(0) /\left(2 u^{\prime 2}\right)$ and $L_{u}^{*}=\pi E_{11}^{*}(0) /\left(2 u^{\prime 2}\right)$, where the superscript + and $*$ indicates the use of the von Kármán spectrum model and the $G$-function model, respectively. The Taylor microscale $\lambda=u^{\prime} /\left(\overline{(d u / d x)^{2}}\right)^{1 / 2}$ is calculated as $\tilde{\lambda}$, where the velocity gradient is calculated from the filtered fluctuating velocity signal, and $\lambda^{*}$, where the velocity gradient is calculated by integrating the G-model corrected spectrum. The dissipation is calculated as $\epsilon_{i s o}=15 \nu \overline{(d u / d x)^{2}}$, and $\epsilon_{X W}=3 \nu \overline{(d u / d x)^{2}}+6 \nu \overline{(d v / d x)^{2}}$. Velocity gradients are calculated either directly from the filtered fluctuating velocity samples (marked by tilde hat) or by integrating the $G$-function model corrected spectrum (marked by superscript $*)$, such that $\overline{(d u / d x)^{2}}=\int k^{2} E_{11}^{*}(k) d k$, and $\overline{(d v / d x)^{2}}=\int k^{2} E_{22}^{*}(k) d k$, where $E_{11}^{*}(k)$ and $E_{22}^{*}(k)$ are calculated using the Taylor hypothesis assuming the local convection velocity equals to the local mean streamwise velocity $U$. The last column is reproduced from Valente and Vassilicos [27] for comparison.

\begin{tabular}{|c|c|c|c|c|c|c|c|c|c|}
\hline \multirow{3}{*}{$\begin{array}{c}\text { Data set } \\
x / x_{\text {peak }} \\
U_{\infty}(\mathrm{m} / \mathrm{s})\end{array}$} & \multicolumn{2}{|c|}{ Honda } & \multicolumn{3}{|c|}{ Lille } & \multicolumn{3}{|c|}{$3 \times 3$} & \multirow{2}{*}{$\begin{array}{c}\text { Valente11 } \\
1.04\end{array}$} \\
\hline & 1 & 1.54 & 1 & 1.65 & 2.73 & 1 & 1.48 & 2.62 & \\
\hline & 10 & 10 & 9 & 9 & 9 & 10 & 10 & 10 & 15 \\
\hline$\tilde{u^{\prime}} / U_{\infty}$ & 0.152 & 0.118 & 0.167 & 0.111 & 0.073 & 0.149 & 0.121 & 0.080 & 0.053 \\
\hline$u^{\prime *} / U_{\infty}$ & 0.145 & 0.117 & 0.166 & 0.110 & 0.072 & 0.149 & 0.121 & 0.079 & - \\
\hline$L_{u}^{+}(\mathrm{m})$ & 0.26 & 0.23 & 0.19 & 0.18 & 0.22 & 0.08 & 0.08 & 0.10 & 0.05 \\
\hline$L_{u}^{*}(\mathrm{~m})$ & 0.30 & 0.26 & 0.20 & 0.19 & 0.23 & 0.08 & 0.08 & 0.10 & - \\
\hline$\tilde{\eta}(\mathrm{mm})$ & 0.17 & 0.19 & 0.16 & 0.19 & 0.25 & 0.12 & 0.14 & 0.18 & 0.15 \\
\hline$\eta^{*}(\mathrm{~mm})$ & 0.17 & 0.19 & 0.14 & 0.18 & 0.24 & 0.12 & 0.13 & 0.18 & - \\
\hline$\tilde{\lambda}(\mathrm{mm})$ & 11.5 & 10.9 & 9.6 & 9.0 & 10.2 & 5.8 & 5.7 & 6.9 & 4.8 \\
\hline$\lambda^{*}(\mathrm{~mm})$ & 10.6 & 10.3 & 7.8 & 8.0 & 9.6 & 5.4 & 5.4 & 6.5 & - \\
\hline$\tilde{R e}_{\lambda}$ & 1161 & 849 & 949 & 593 & 443 & 570 & 458 & 364 & 253 \\
\hline$R e_{\lambda}^{*}$ & 1015 & 802 & 773 & 522 & 414 & 532 & 431 & 341 & - \\
\hline$\tilde{\epsilon}_{i s o}\left(\mathrm{~m}^{2} \mathrm{~s}^{-3}\right)$ & 3.9 & 2.7 & 5.6 & 2.8 & 0.9 & 15.1 & 10.1 & 3.0 & 6 \\
\hline$\tilde{\epsilon}_{X W}\left(\mathrm{~m}^{2} \mathrm{~s}^{-3}\right)$ & - & - & - & - & - & 9.2 & 6.2 & 1.9 & 4.7 \\
\hline$\epsilon_{i s o}^{*}\left(\mathrm{~m}^{2} \mathrm{~s}^{-3}\right)$ & 4.6 & 2.9 & 8.3 & 3.5 & 1.0 & 17.3 & 11.3 & 3.3 & - \\
\hline$\epsilon_{X W}^{*}\left(\mathrm{~m}^{2} \mathrm{~s}^{-3}\right)$ & - & - & - & - & - & 10.5 & 7.0 & 2.1 & - \\
\hline
\end{tabular}

Another issue is the validity of the isotropic assumption used in calculating the dissipation rates. Comparisons are made between $\epsilon_{i s o}=15 \nu \overline{(d u / d x)^{2}}$ and $\epsilon_{X W}=3 \nu \overline{(d u / d x)^{2}}+6 \nu \overline{(d v / d x)^{2}}$ from the $3 \times 3$ experiments. To calculate the dissipation rate $\epsilon_{X W}^{*}$ from the spectrum, it is assumed that $\overline{(d u / d x)^{2}}=\int k^{2} E_{11}^{*}(k) d k$, and $\overline{(d v / d x)^{2}}=\int k^{2} E_{22}^{*}(k) d k$, where $E_{11}^{*}(k)$ and $E_{22}^{*}(k)$ are calculated using the Taylor hypothesis assuming a local convection velocity equal to the local mean streamwise velocity $U$ (as justified in the beginning of section II C). It seems that the isotropy assumption causes an overestimation of the values of $\epsilon_{i s o}$, and the difference is roughly $\pm 24 \%$ at $x / x_{\text {peak }}=1$, whereas Valente and Vassilicos [27] give a $\pm 12 \%$ difference for the square fractal grid at $x / x_{\text {peak }}=1.04$. Note, however, the value of $\epsilon_{X W}$ might be underestimated for cross wires since the $1 \mathrm{~mm}$ separation of the two wires is approximately $10 \eta$ [see 27 ]. The legitimacy of the isotropy assumption is further discussed in section III D regarding the study of dissipation.

\section{RESULTS}

In this section the results are presented in the following manner. First of all, the Reynolds number effect of the wake of the grid bars is examined using the Honda experiment data. The wake interaction length scale is then examined using the streamwise evolution of turbulence statistics. Observations on the evolution of turbulence length scales are presented in section III C, followed by discussions on the homogeneity, Gaussianity and isotropy of the flow. Finally, the non-equilibrium scaling relation for current turbulent flows are examined using the dissipation coefficient and spectra. 

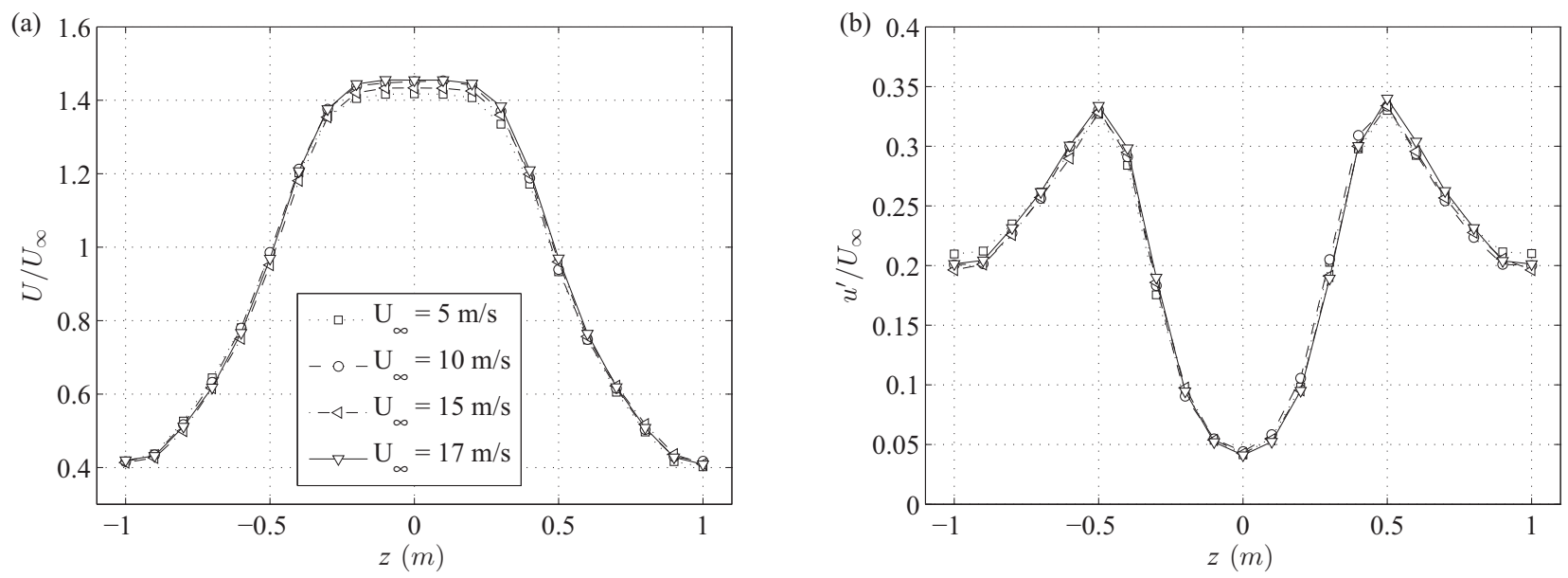

FIG. 7. Transverse profiles of (a) mean velocity, and (b) turbulence intensity from the Honda experiments, at different inlet velocities $U_{\infty}$ at $x / x_{\text {peak }}=0.38, y=0 \mathrm{~m}$.

\section{A. Reynolds number effect}

The transverse velocity profiles leeward of the grid center (figure 7 a) strongly depends on the wake generated by the largest grid bars. It is therefore important to understand the Reynolds number effect of the turbulent wakes. For that reason, the flow is probed across the span at $x=2 \mathrm{~m}$ (or $\left.x / x_{\text {peak }}=0.38\right) y=0 \mathrm{~m}$ at different Reynolds numbers $R e_{0}=U_{\infty} L_{0 y} / \nu=6.5 \times 10^{5}$ to $1.9 \times 10^{6}$.

Figure 7 gives the results for the mean velocity and turbulence intensity profiles at $U_{\infty}=5,10,15,17 \mathrm{~m} \mathrm{~s}^{-1}$. It is obvious that the normalization by inlet velocity $U_{\infty}$ collapses both mean velocity and turbulence intensity profiles. This suggests that the shape of the wake is independent of the inlet Reynolds number $R e_{0}$.

\section{B. Wake interaction length scales}

The wake interaction length scale $x_{*}^{\text {peak }}$ was originally introduced by Gomes-Fernandes et al. [18] to characterize the location where the wakes generated by the largest grid bars meet, and this length scale successfully collapsed turbulence intensity profiles from several experiments with FSG. It is defined as $x_{*}^{\text {peak }}=0.21 L_{0}^{2} /\left(\alpha C_{d} t_{0}\right)$, which incorporates the incoming flow turbulence condition via the constant $\alpha$, the length $L_{0}$, the width $t_{0}$, and the drag coefficient $C_{D}$ of the largest grid element.

Note though, the notation $x_{*}^{\text {peak }}$ is taken after Gomes-Fernandes et al. [18] for consistency. In the original paper this wake interaction length scale physically gives the turbulence intensity peak location $x_{\text {peak }}$ such that $x_{\text {peak }}=x_{*}^{\text {peak }}$, but this is not the case for the current study, as the actual turbulence intensity peak location is further downstream such that $x_{\text {peak }}>x_{*}^{\text {peak }}$. One should not confuse $x_{*}^{\text {peak }}$ with the actual turbulence intensity peak location $x_{\text {peak }}$. Since the RFG consist of two distinct large scales, the wake interaction length-scale can be calculated in either direction, as shown in table III. For the current discussion, $x_{z *}^{p e a k}$ is used to normalize the streamwise development as it is assumed that the thickest vertical bars generate most of the turbulent energy. The result is shown in figure 8 together with profiles reproduced from Mazellier and Vassilicos [17] at $U_{\infty}=5.2 \mathrm{~m} / \mathrm{s}$.

From figure 8 , it can be observed that the length scale $x_{z *}^{p e a k}$ fails to collapse the data from FSGs and RFGs. All of the turbulence intensity profiles from Mazellier and Vassilicos [17] peak at $x / x_{*}^{\text {peak }}=1$ as expected, but for the RFGs, this peak location is at $x / x_{z *}^{\text {peak }}=1.40$, which corresponds to $x / x_{y *}^{\text {peak }}=1.53$.

This discrepancy can be explained by a review of the scaling proposed by Gomes-Fernandes et al. [18], who followed the results from Symes and Fink [20] to derive their scaling for the evolution of the wake half width and intensity. 


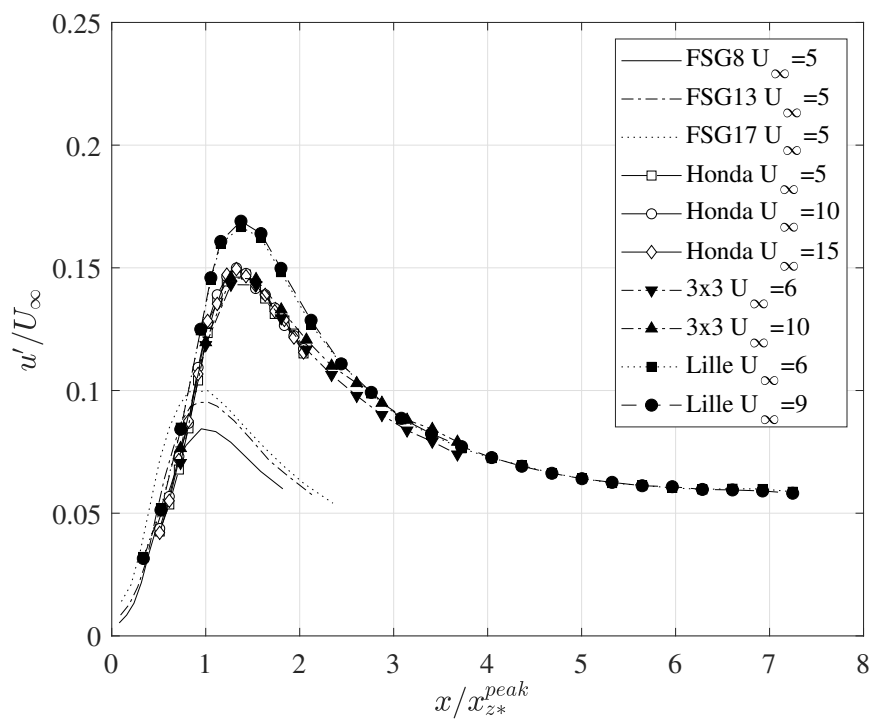

FIG. 8. Turbulence intensity profiles at centerline versus $x / x_{z *}^{p e a k}$ from all experiments. FSG data reproduced from Mazellier and Vassilicos [17].
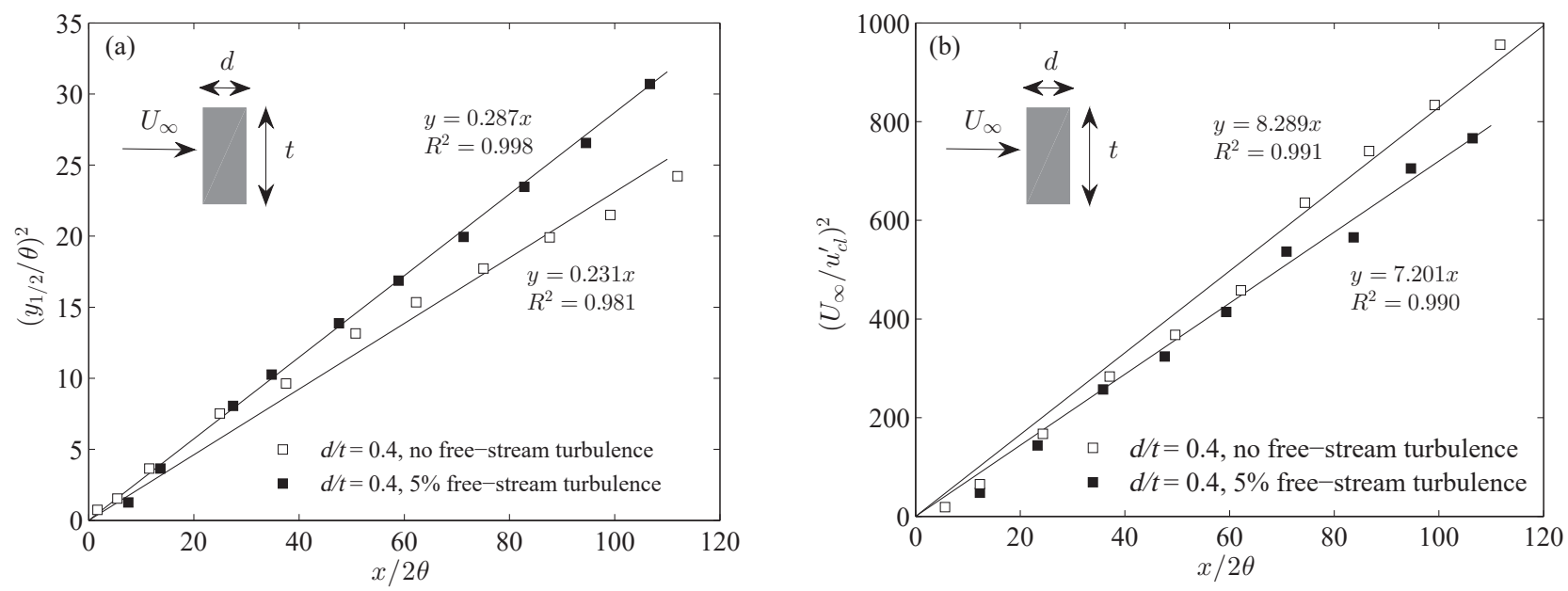

FIG. 9. Influence of external turbulence on the development of (a) wake half-width, and (b) turbulence intensity. Reproduced after Symes and Fink [20], where $R^{2}$ is the coefficient of determination such that $R^{2}=1$ indicates a perfect fit with no residual error.

The effect of the free stream turbulence was studied, as reproduced in figure 9 , and was characterized by the constant coefficients $\alpha$ and $\beta$ as in

$$
\left(\frac{y_{1 / 2}}{\theta}\right)^{2}=\alpha \frac{x}{2 \theta}, \quad\left(\frac{U_{\infty}}{u_{c l}^{\prime}}\right)^{2}=\beta \frac{x}{2 \theta},
$$

where $y_{1 / 2}$ is the wake half width, $\theta$ is the momentum thickness, and $u_{c l}^{\prime}$ is the turbulence intensity at the centerline of the wake. The parameters $\alpha$ and $\beta$ are functions of the free stream turbulence intensity and were used by GomesFernandes et al. [18] in their derivation of $x_{*}^{\prime}=L_{0}^{2} /\left(\alpha C_{D} t_{0}\right)$ assuming that $L_{0}=2 y_{1 / 2}$ and $2 \theta=C_{D} t_{0}$. The final 

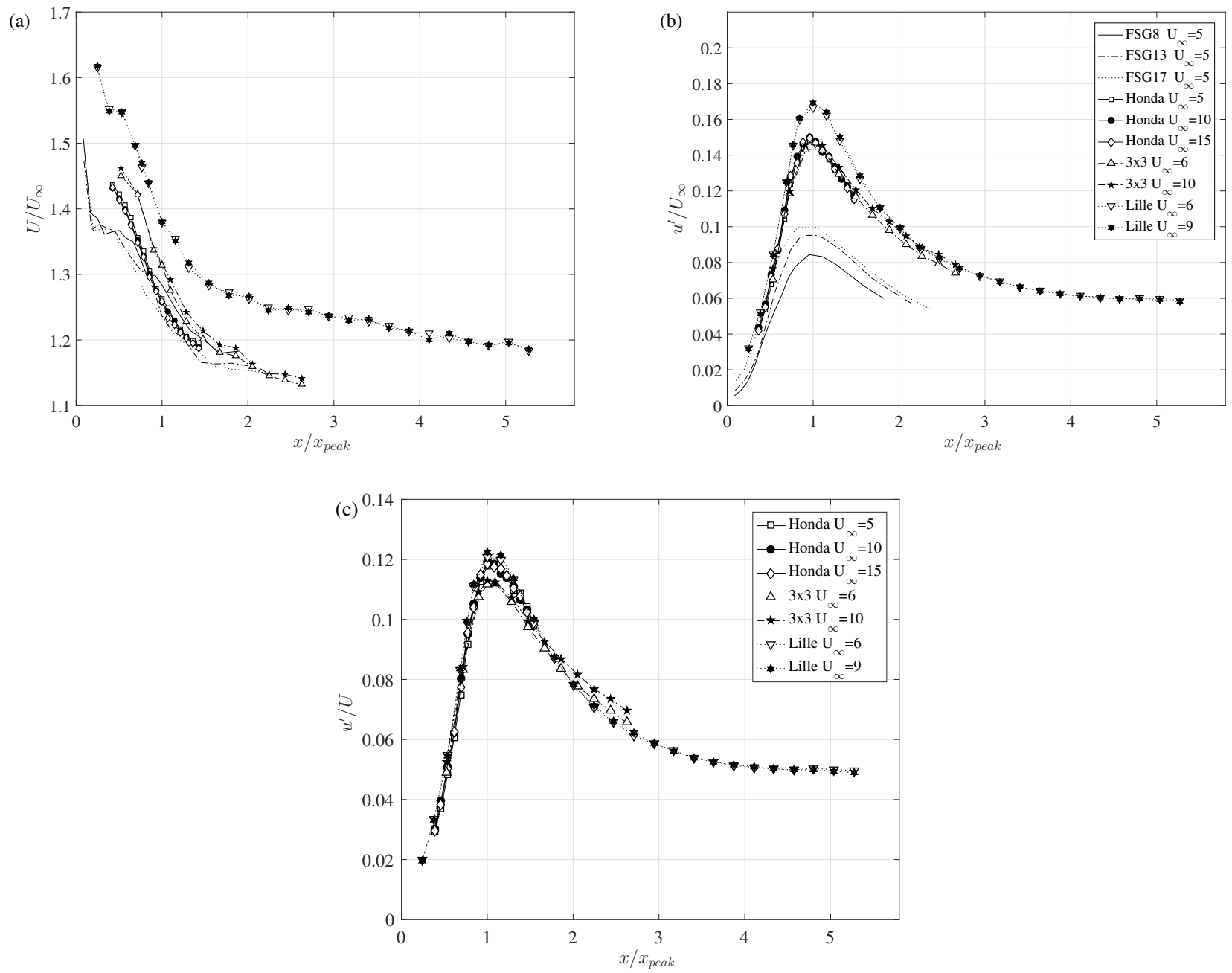

FIG. 10. (a) Mean streamwise velocity profiles normalized by $U_{\infty}$, (b) turbulence intensity profiles normalized by $U_{\infty}$, and (c) turbulence intensity profiles normalized by local mean velocity $U$ along the centerline against $x / x_{\text {peak }}$ from all experiments. The data from fractal square grids (FSG) is reproduced from Mazellier and Vassilicos [17].

definition of $x_{*}^{\text {peak }}=\phi x_{*}^{\prime}$ incorporates a coefficient $\phi=0.21$ to scale the peak turbulence intensity at $x / x_{*}^{\text {peak }}=1$, and the value was determined empirically based on results from Mazellier and Vassilicos [17].

The spreading rate of wake depends not only on the free stream turbulence, but also on initial/inlet conditions, as suggested by Wygnanski et al. [51] and George [52]. The reason for the discrepancies evident in figure 8 lies most likely in the shape of the grid. Firstly, the spreading rate might be affected by the local shear on the grid plane due to the non-uniform grid geometry. This is also evident from equation (7) as suggested by the inclusion of the momentum thickness $\theta$, which will be affected by the presence of a mean shear. Secondly, there are two distinctive wake interaction length-scales in the flow, and the wakes from both directions collectively affect the actual turbulence peak location. These effects cannot be quantified at the moment, but for a given ratio of the largest lengths of the grid bars, i.e. $L_{0 z} / L_{0 y}$, the value of the coefficient $\phi$ should be the same. That is to say, equation (3) needs to be generalized such that $x_{*}^{\text {peak }}=\phi L_{0 z}^{2} /\left(\alpha C_{d} t_{0 z}\right)$, where $\phi=f\left(L_{0 z} / L_{0 y}\right)$ (e.g. $\phi=f(1.945)=0.294$ for the current grid geometry), to calculate the actual location of peak turbulence intensity. For the rest of the discussion, the measured physical turbulence peak location $x_{\text {peak }}$ is used to normalize the streamwise distance for simplicity. 

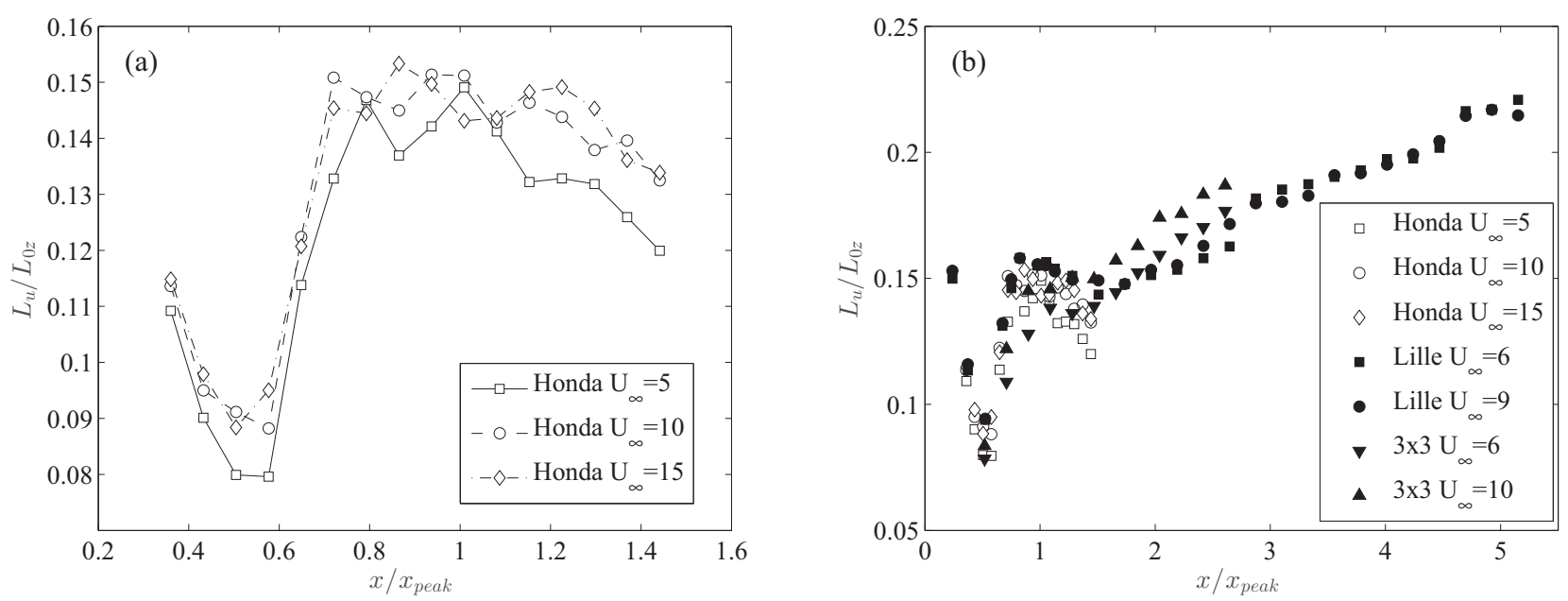

FIG. 11. Longitudinal integral length scale profiles $L_{u} / L_{0 z}$ along the centerline from (a) the Honda experiment, and (b) all three experiments.

The streamwise mean velocity and turbulence intensity profiles for all experiments are given in figure 10. The mean profiles collapse well except for the Lille experiments, where both the mean velocity and turbulence intensity are notably higher. The difference in the mean velocity might be explained by the different measurement methods of $U_{\infty}$. A Pitot tube is used in the Lille experiments (roughly $10 \mathrm{~cm}$ off the centerline and $20 \mathrm{~cm}$ upstream of the grid), and wall pressure across the contraction is used in the other two experiments. Therefore, the exact incoming velocity $U_{\infty}$ is slightly different due to the non-uniform grid geometry. This effect is also seen in figure 10 (b). In fact, when the turbulence intensity is normalized with the local mean velocity $U$, the collapse from all experiments clearly improves (figure 10 (c), and the discrepancies are within $1 \% U$.

\section{Integral length scales and Taylor microscales}

To further understand the streamwise evolution of the turbulence characteristics, the length scales of the turbulence are of interest. All of the length scales are calculated from the corrected spectra, as discussed in section II C, and the superscript $*$ is therefore omitted henceforth, such that $L_{u} \equiv L_{u}^{*}$. Previously, it has been shown that the longitudinal integral length scale $L_{u}$ (i.e. $L_{u}=L_{u u, x}$, the streamwise integral length scale of fluctuating velocity $u$ ) in the FSGgenerated turbulence has a magnitude of approximately $0.2 L_{0}$ in the decay region $\left(x / x_{\text {peak }}>1\right)$, where $L_{0}$ is the length of the largest grid bar, and that the ratio of $L_{u} / \lambda$ remains roughly constant in a significant part of the decay region $[17,18,29]$.

The integral length scale $L_{u}$ measured from the Honda experiments is given in figure 11 (a), where the length scale $L_{u} / L_{0 z}$ appears to decrease in the decay region from $x / x_{\text {peak }}=1$ to 1.5 . This is surprising as the integral length scale is expected to grow in decaying turbulence, or remain roughly constant in case of the non-equilibrium dissipation. Nevertheless, the decreasing behavior is confirmed by the results from the $3 \times 3$ and Lille experiments, as shown in figure 11 (b), which also reveal that this decrease exists only in the beginning of the decay region. The value of $L_{u}$ increases monotonically after $x / x_{\text {peak }}=1.5$.

Note that $L_{u}$ in figure 11 is normalized as $L_{u} / L_{0 z}$, and this ratio is doubled if $L_{u} / L_{0 y}$ is used. In the region from $x / x_{\text {peak }}=1$ to 1.5 , the average value of $L_{u} / L_{0 z}$ is approximately 0.14 , or equivalently $L_{u} / L_{0 y} \approx 0.28$. To draw comparison between FSG and RFG, we refer to square and rectangular wind tunnel cross sections, respectively. The corresponding length scale of the grid bar of RFG and FSG in a rectangular wind tunnel yields $L_{0 y} \approx L_{0}\left(L_{0}\right.$ being the largest bar length in FSG grids), while in square wind tunnel it is $L_{0 z} \approx L_{0}$. As such, the largest integral length scales produced by the RFG and FSG are compared between $L_{u} / L_{0 y} \approx 0.28$ and $L_{u} / L_{0} \approx 0.2$ for rectangular wind tunnels, or $L_{u} / L_{0 z} \approx 0.14$ versus $L_{u} / L_{0} \approx 0.2$ for square wind tunnels. Therefore, it can be concluded that the grid geometry 

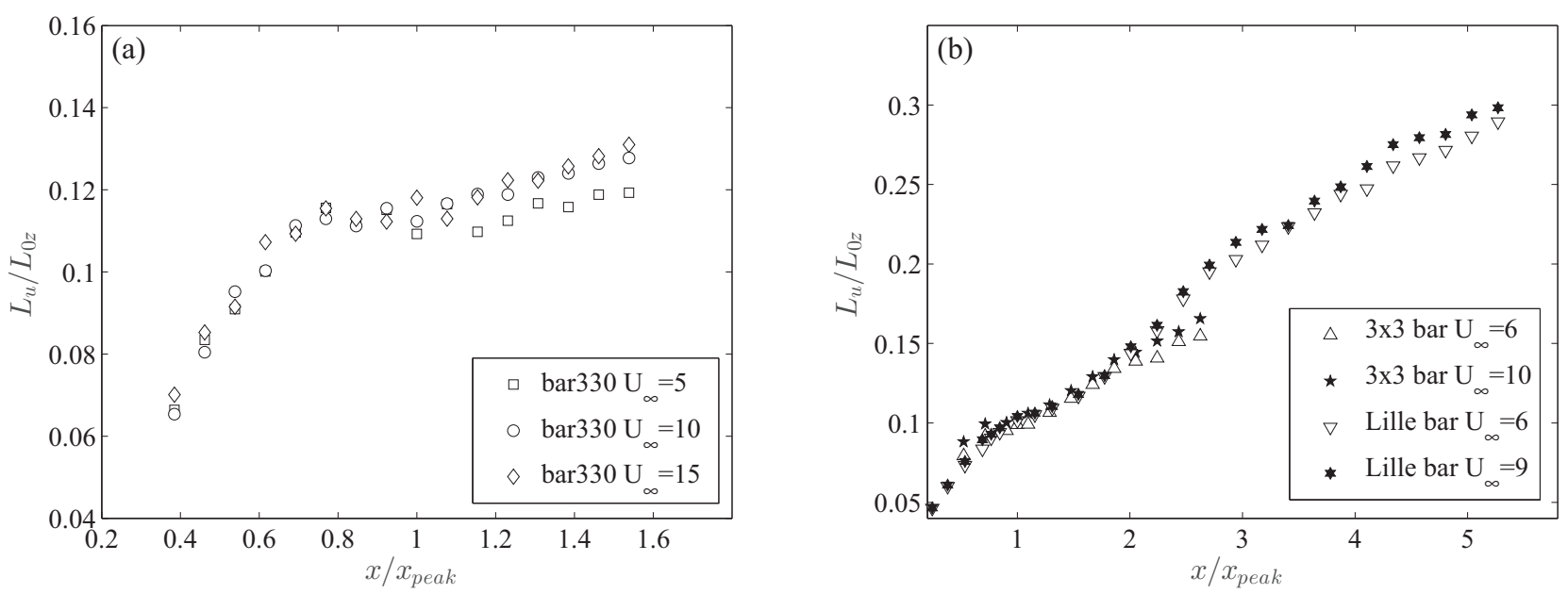

FIG. 12. Longitudinal integral length scale profiles $L_{u} / L_{0 z}$, (a) from the Honda experiment along the streamwise direction at $y=0.33 \mathrm{~m}$, and (b) from the $3 \times 3$ and Lille experiments behind the center of the horizontal bar.

matching the shape of wind tunnel cross section would produce the largest possible integral length scales. Since most wind engineering wind tunnels incorporate rectangular cross sections, the RFG does offer an option to produce larger integral length scales for engineering applications. Furthermore, with the regional decrease of $L_{u}$ (figure 11) and monotonic decreasing turbulence intensity (figure 10), various combinations of length scale and turbulence intensity might be realized by choosing the desired streamwise location $x / x_{\text {peak }}$.

The streamwise profiles of $L_{u}$ with $y$ offsets are shown in figure 12. The Honda case shows the streamwise profile at $y=0.33 \mathrm{~m}$, which is approximately at the height of the smallest grid bars closest to the center. It seems that the value of $L_{u}$ decreases only slightly in the region from $x / x_{\text {peak }}=0.8$ to 1 before monotonically increasing afterwards. All of the profiles behind horizontal bars from the $3 \times 3$ and Lille experiments in figure 12 (b) show a monotonic increase throughout the measurement domain. The increasing rate seems impeded slightly in the region of $0.8<x / x_{\text {peak }}<1.5$, but is recovered immediately at $x / x_{\text {peak }}>1.5$. This observation might suggest that the decrease of the length scale is caused by the interaction of the two vortex systems from the horizontal and vertical bars. Such interaction is strongest at the centerline, and diminishes with increasing offset, which agrees with the observations.

To confirm the decrease of integral length scale in the region $1<x / x_{\text {peak }}<1.5$, two-point correlations are also measured at $x=6 \mathrm{~m}$ and $8 \mathrm{~m}$, or $x / x_{\text {peak }}=1.15$ and 1.54 in the Honda experiments. The values of $L_{u u, y}$ are calculated by integrating up to the first zero-crossing point, which shows a decrease from $L_{u u, y} \approx 116 \mathrm{~mm}$ (at $\left.x / x_{\text {peak }}=1.15\right)$ to $L_{u u, y} \approx 111 \mathrm{~mm}\left(\right.$ at $\left.x / x_{\text {peak }}=1.54\right)$. The results support our discussion of the decreasing integral length scale $L_{u}$ in the beginning of the decay region.

The evolution of the Taylor microscale is given in figure 13 for each experiment with streamwise profiles along the centerline and along the streamwise direction with $y$ offset. The Taylor microscale $\lambda$ is calculated by $\lambda=\lambda^{*}=$ $u^{\prime} /\left(\overline{(d u / d x)^{2}}\right)^{1 / 2}$, where the velocity gradient is calculated by integrating the G-model corrected spectrum assuming Taylor's hypothesis. It shows a similar trend to the integral length scales, where their values decrease in the region of $x / x_{\text {peak }}=1$ to 1.5 along the centerline, and grow monotonically elsewhere. The value of $\lambda$ at two $y$ locations becomes similar after approximately $x / x_{\text {peak }}=1.8$.

These observations agree with the conclusion that the decrease of length scales is caused by the wake interaction, which is strongest on the centerline at $x / x_{\text {peak }}=1$, and diminishes away from this location. This decrease of turbulence length scales is observed for the first time in decaying turbulence, and the exact mechanism perhaps calls for another study of the flow structures in the region around $x / x_{\text {peak }}=1$ using, for example, Particle Image Velocimetry, to fully understand the dynamics. 

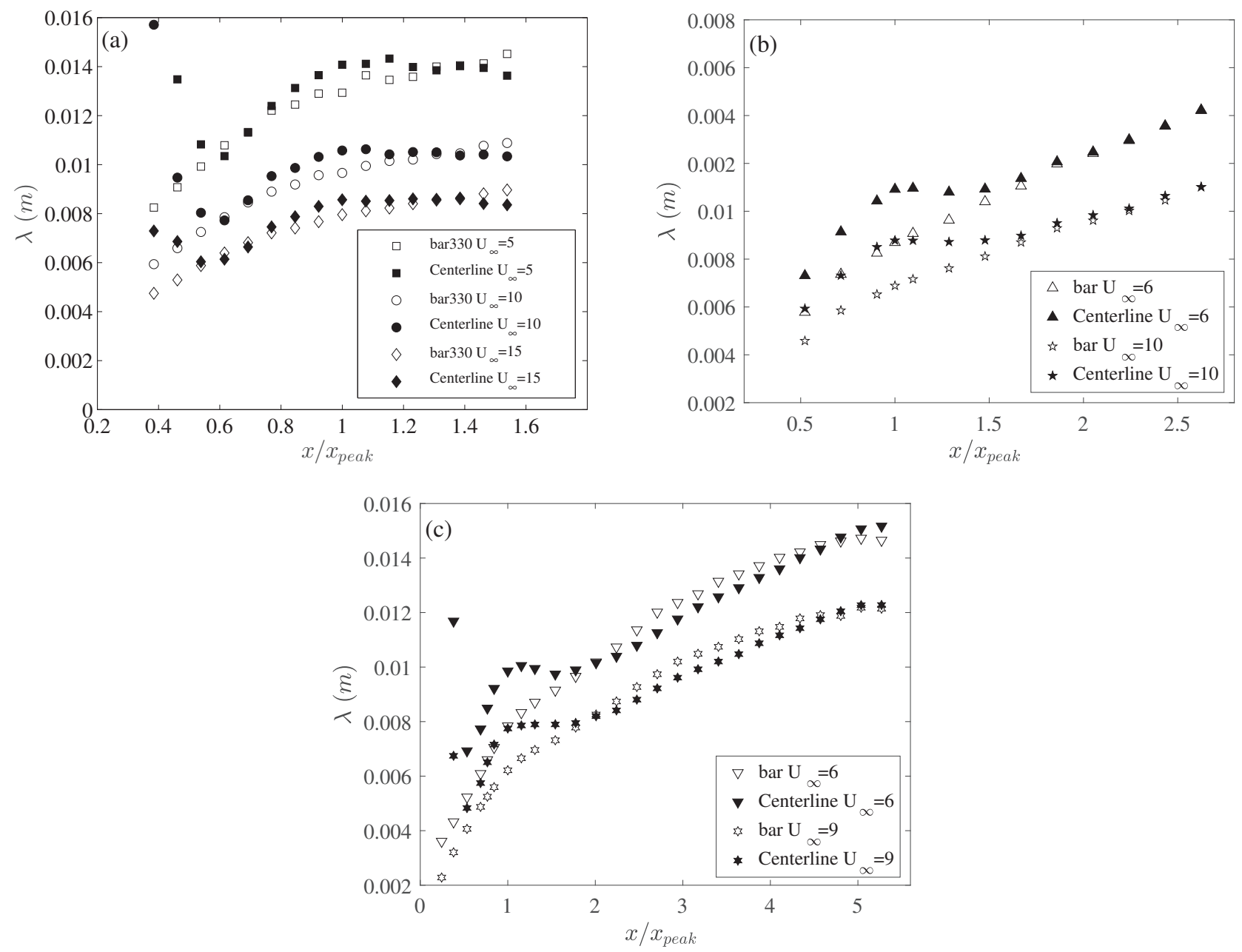

FIG. 13. Streamwise profiles of the Taylor microscale $\lambda$ from (a) Honda experiments, (b) $3 \times 3$ experiments, and (c) Lille experiments.

\section{Homogeneity, Gaussianity, and isotropy}

The homogeneity of the RFG-generated turbulent flow is assessed using the mean statistics from the Honda wind tunnel experiments. The transverse profiles at different $y$ locations and the two-dimensional assembly contours are given in figures 14 and 15 . At $x=2 \mathrm{~m}\left(x / x_{\text {peak }}=0.38\right)$, the mean velocity contour was plotted assuming $y$ symmetry of the flow, and it suggests that there is a rectangular jet-like structure in the middle of the grid with higher mean velocities. At $x=8 \mathrm{~m}\left(x / x_{\text {peak }}=1.54\right)$, the flow was measured across half of the span and symmetry was assumed in both $y$ and $z$ directions to produce figure 14 (b). The variations are much reduced, which suggests that the flow is approaching a homogeneous state. The velocity contour in figure 14 (b) show an oval shape at $x / x_{\text {peak }}=1.54$, in contrast to the rectangular shape at $x / x_{\text {peak }}=0.38$. This is different from the FSG results where axisymmetric shapes are observed immediately in the decay region [53-55] due to the fact that $L_{0 y} \neq L_{0 z}$.

The profiles and the assembled contours of the turbulence intensity fields are given in figure 15 . The turbulence intensity has its minimum around the centerline, and it peaks just off the side of the biggest bars, where the gradient of the mean velocity is largest as seen in figure 14. This peak location moves aside with increasing height corresponding to the shape of the wake, which is altered by the smaller iterations on the grid. In general, the turbulence intensity 

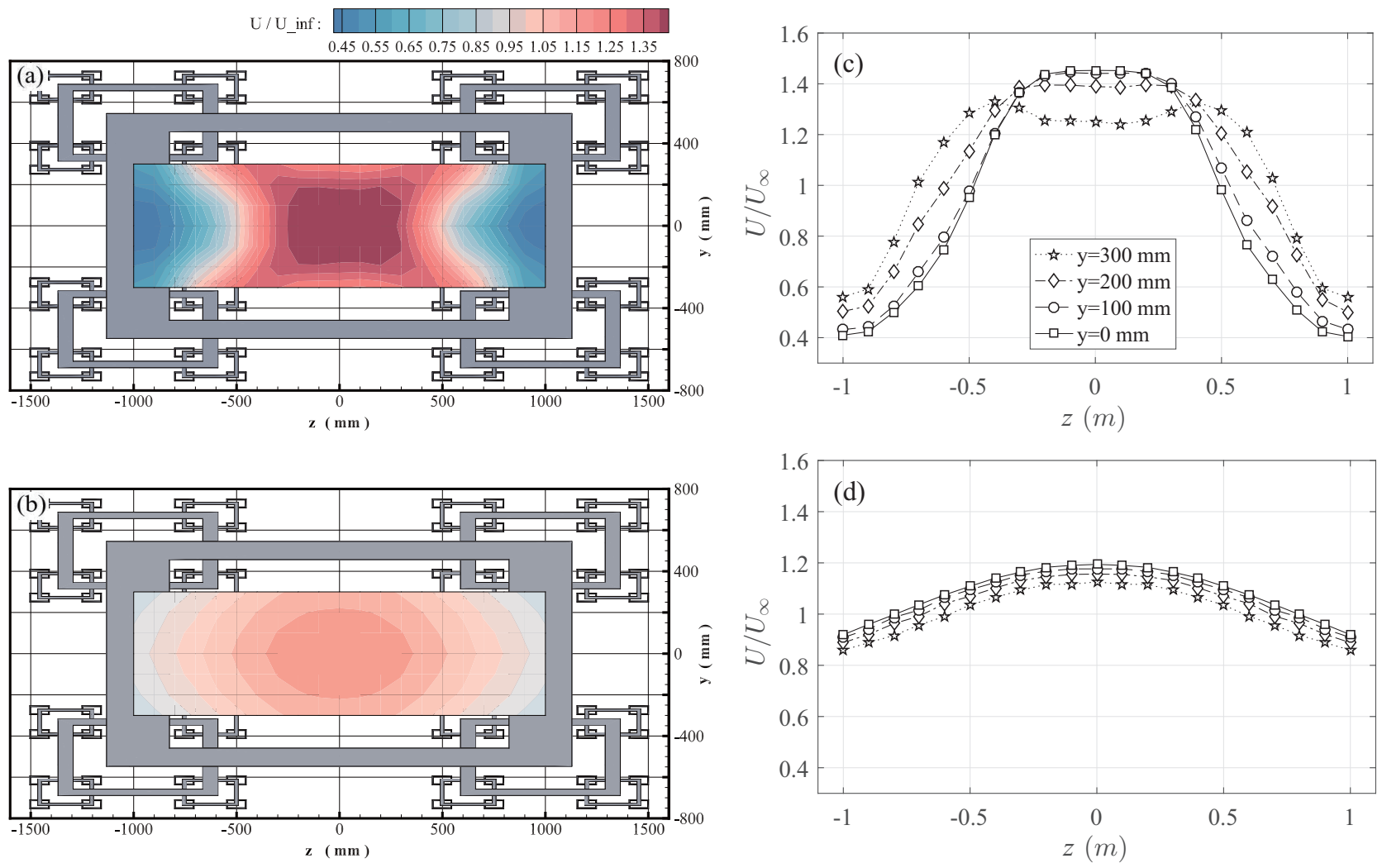

FIG. 14. Contours of $U / U_{\infty}$ at $x / x_{\text {peak }}=0.38$ (a), and $x / x_{\text {peak }}=1.54$ (b) at $U_{\infty}=10 \mathrm{~m} / \mathrm{s}$ from the Honda experiments, assembled from (c) and (d), respectively.

profiles becomes more homogeneous with increasing $y$, which seems to support the idea that the smaller iterations tend to homogenize the flow. At $x / x_{\text {peak }}=1.54$, the variation of $u^{\prime} / U_{\infty}$ across the span is reduced to approximately $3 \%$, as shown in figure 15 .

To further quantify the homogeneity in the streamwise direction, we refer to the criteria proposed by Corrsin [56], namely

$$
\begin{gathered}
\partial L_{u} / \partial x \ll 1, \\
\left(L_{u} / \lambda\right) \partial \lambda / \partial x \ll 1, \\
\left(L_{u} / \overline{u^{2}}\right) \partial \overline{u^{2}} / \partial x \ll 1 .
\end{gathered}
$$

The results for these relations from all experiments are presented in figure 16. The values of $\partial L_{u} / \partial x$ and $\left(L_{u} / \lambda\right) \partial \lambda / \partial x$ are all approximately 0 in the decay region. This do not contradict the increasing integral length scales observed in figure 11 and 12, but rather implies that the variation is indeed slow. The results for $\left(L_{u} / \overline{u^{2}}\right) \partial \overline{u^{2}} / \partial x$, however, show a larger deviation from 0 . The minimum appears to occur at $x / x_{\text {peak }} \approx 1.5$, which corresponds to the region of decreasing length scales. The maximum deviation is approximately 0.075 , which is larger than the reported value of 0.05 from the FSG generated turbulence [29]. The results along the streamwise direction with $y$ offsets are also given in figure 16, which appear similar to that along the centerline, and similarly the most obvious deviation from homogeneity is observed in figure 16 (f). These results seem to suggest that the turbulence in the beginning of the decay region $1<x / x_{\text {peak }}<1.5$ is not strictly homogeneous, but the rest of the decay region might be considered homogeneous under the criteria of relations 8.

The Gaussianity of the flow was also examined using the probability density functions $(p d f)$ of the streamwise 

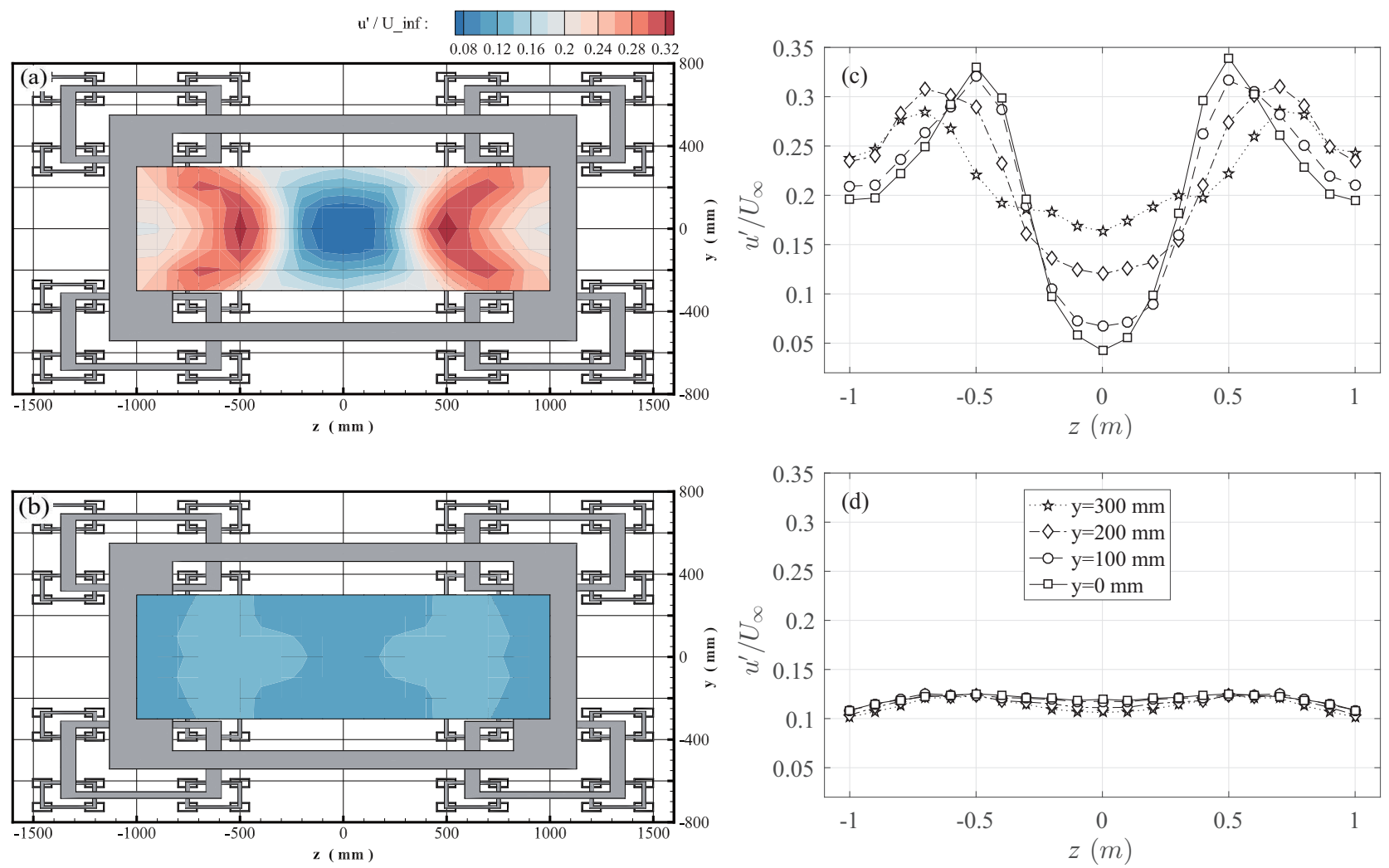

FIG. 15. Contours of $u^{\prime} / U_{\infty}$ at $x / x_{\text {peak }}=0.38$ (a), and $x / x_{\text {peak }}=1.54$ (b) at $U_{\infty}=10 \mathrm{~m} / \mathrm{s}$ from the Honda experiments, assembled from (c) and (d), respectively.

fluctuating velocity. Figure 17 (a) shows the $p d f$ of $u / u^{\prime}$ at the peak location $x / x_{\text {peak }}=1$ from different experiments with different inlet Reynolds numbers. The collapse of the $p d f$ confirms the independence on inlet Reynolds number. The distribution is clearly non-Gaussian, and skewed to the left, which means more negative velocity fluctuations, depicting a negative velocity skewness $S_{u}$. From the $3 \times 3$ experiments, the $p d f$ from different streamwise locations along the centerline are shown in figure 17 (b). The $p d f$ approached to Gaussian in the decay region at $x / x_{\text {peak }}=1.4$ and $x / x_{\text {peak }}=2.6$, with the latter being slightly better. As demonstrated in figure 17 (c), the $p d f$ of $u / u^{\prime}$ beyond $x / x_{\text {peak }}=2.6$ from the Lille experiments are skewed to the left again, suggesting the deviation away from Gaussianity further downstream for the Lille experiment. Nonetheless, this part of data from the Lille experiment, where the development might be interfered by the growing boundary layer from the wall, is included for the discussion as it serves as a reference for possible applications to use RFGs in wind engineering studies.

To look at the isotropy of the flow, we use the data from the $3 \times 3$ experiments where both $u$ and $v$ components of the velocity were measured. Ideally, large scale isotropy of turbulent flow requires $u^{\prime} / v^{\prime}=u^{\prime} / w^{\prime}=1$ and $L_{u} / L_{v}=$ $L_{u} / L_{w}=2$ (where $L_{v}=L_{v v, x}$ is the streamwise integral length scale of fluctuating velocity $v$ ). The results are presented in figure 18. In figure 18 (a), the ratio of $u^{\prime} / v^{\prime}$ behind the centerline and behind the bar are both larger than 1 , and gradually converges to $u^{\prime} / v^{\prime}=1.35$. This value is larger than the previously reported values of $u^{\prime} / v^{\prime}=1.05 \sim 1.15$ [5] for passive bi-plane grid-generated turbulence, $u^{\prime} / v^{\prime}=1.13 \sim 1.16$ [57], $u^{\prime} / v^{\prime}=1.21 \sim 1.43$ [8] for active-gridgenerated turbulence, and $u^{\prime} / v^{\prime}=1.1 \sim 1.25$ for FSG-generated turbulence [27, 29], respectively.

This anisotropy is also evident in figure 18 (b), where the integral length scale ratio $L_{u} / L_{v}$ is everywhere well above 2 on the centerline. This is comparable to the results of Hearst and Lavoie [29], where they reported values of $L_{u} / L_{v}$ of 2.4 to 2.5 in the decay region. These observations of large scale anisotropy seem to come from the two distinct length scales $L_{0 y}$ and $L_{0 z}$, such that the largest eddy scales in these two directions are much different, which makes sense as they are determined by the physical size of the grid bars in each direction. 

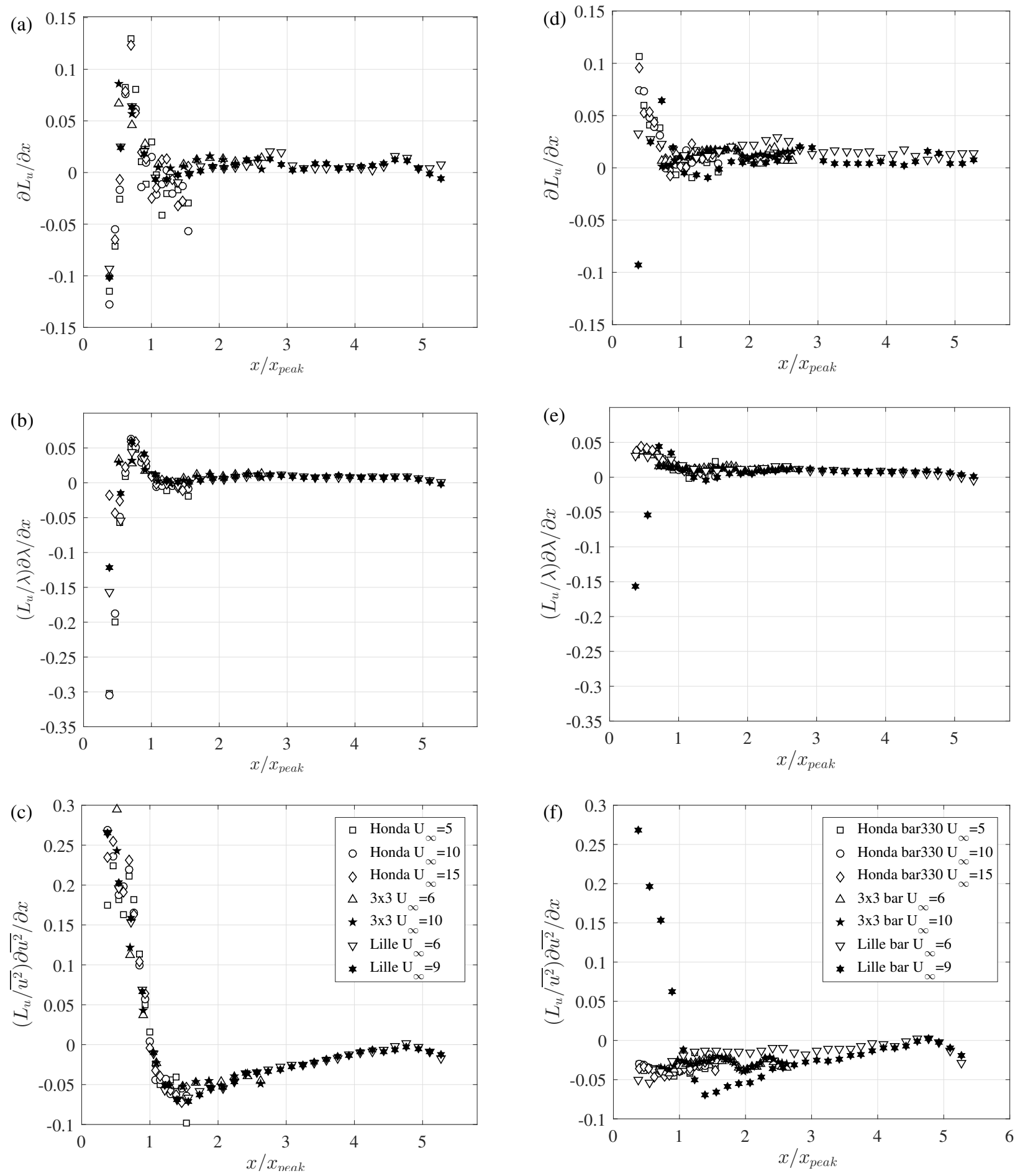

FIG. 16. Streamwise homogeneity of $\partial L_{u} / \partial x(\mathrm{a}, \mathrm{d}),\left(L_{u} / \lambda\right) \partial \lambda / \partial x(\mathrm{~b}, \mathrm{e})$, and $\left(L_{u} / \overline{u^{2}}\right) \partial \overline{u^{2}} / \partial x(\mathrm{c}, \mathrm{f})$ from three experiments. The left column presents the data measured along the centerline. The right column presents the data measured along the streamwise direction with an $y$ offset, i.e. behind the horizontal bar in $3 \times 3$ and Lille experiments (denoted by bar), and at $y=330 \mathrm{~mm}, z=0 \mathrm{~mm}$ in the Honda Experiment (denoted by bar330). 

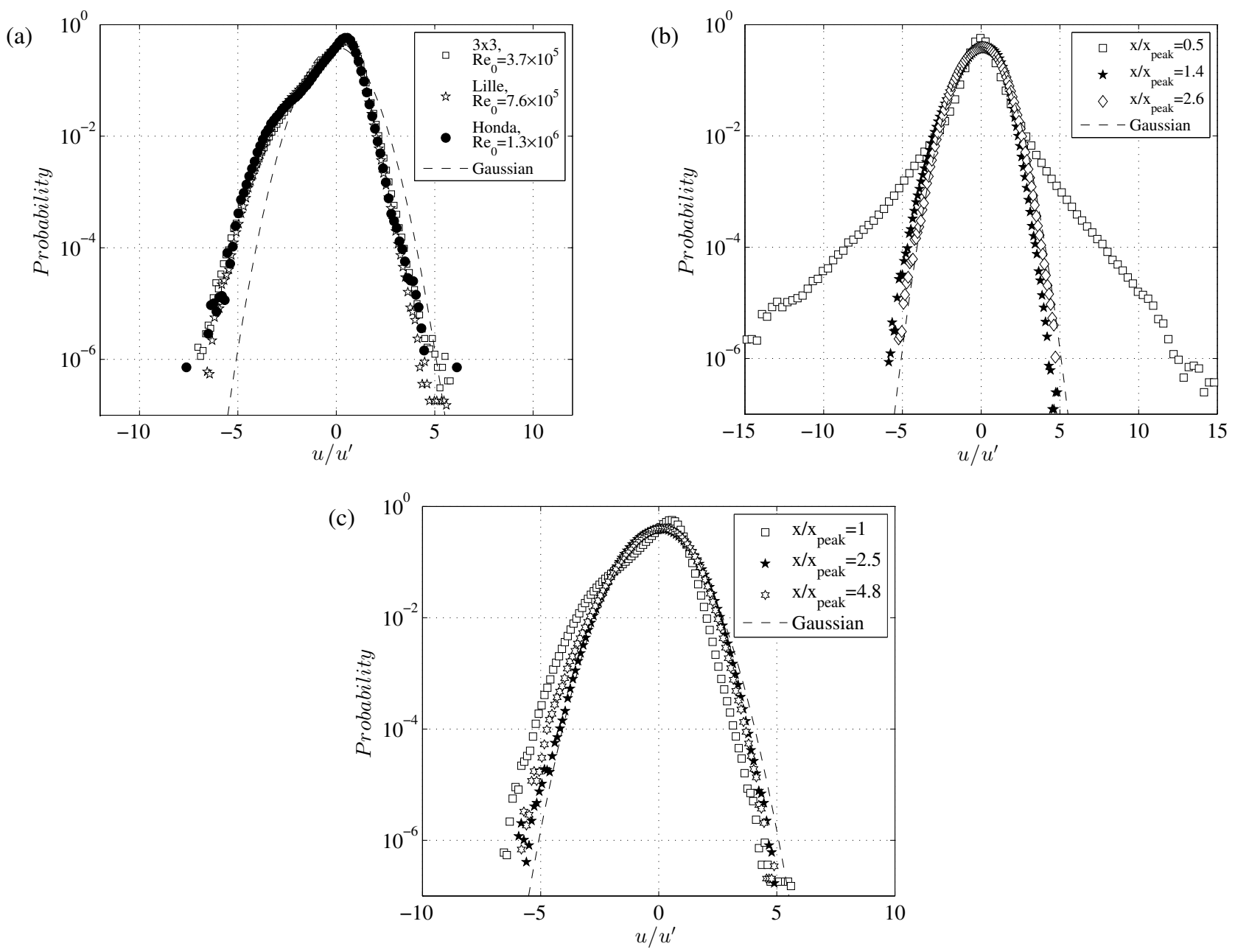

FIG. 17. Probability density function of $u / u^{\prime}$ (a) at $x / x_{\text {peak }}=1$ with different inlet Reynolds numbers $R e_{0}=U_{\infty} L_{0 z} / \nu,($ b) at different streamwise locations along the centerline in the $3 \times 3$ experiment with $U_{\infty}=10 \mathrm{~m} / \mathrm{s}$, and (c) at different streamwise locations along the centerline in the Lille experiment with $U_{\infty}=9 \mathrm{~m} / \mathrm{s}$. The dashed lines give the Gaussian distribution.

The small scale isotropy indicator $\overline{(d v / d x)^{2}} / \overline{(d u / d x)^{2}}$ is shown in figure 19. It is clear that this ratio is much smaller than the isotropic value of 2 , even though the values are increasing monotonically. The values behind the horizontal bars are slightly larger (and hence marginally more isotropic) than that on the centerline, which is in agreement with the previous observation on the large scale isotropy. The ratio of $\overline{(d v / d x)^{2}} / \overline{(d u / d x)^{2}}$ is smaller than that from the FSG-generated turbulence reported by Valente and Vassilicos [27], where they showed $\overline{(d v / d x)^{2}} / \overline{(d u / d x)^{2}} \approx 1.4$ in the decay region. The anisotropy partly explains the discrepancies of the dissipation rate $\epsilon$ calculated with different methods, as discussed in section II C.

Finally, to further evaluate the isotropy assumption in discussing the decaying turbulence, the ratios of the advection and dissipation $-\mathcal{A}_{\text {iso }} / \epsilon_{\text {iso }}$ and $-\mathcal{A}_{X W} / \epsilon_{X W}$ are examined, where $\mathcal{A}_{\text {iso }}=(3 / 2) U\left(\partial \overline{u^{2}} / \partial x\right)$, assuming $\overline{u^{2}}=\overline{v^{2}}=\overline{w^{2}}$, and $\mathcal{A}_{X W}=U(\partial k / \partial x)$, where $k=\left(\overline{u^{2}}+2 \overline{v^{2}}\right) / 2$. Results from the $3 \times 3$ experiments are given in figure 20 (a). It can be seen that the ratio roughly collapses for both cases with and without the isotropy assumption, and stays roughly constant in the region $x / x_{\text {peak }}>1.1$. This is slightly different from the results discussed by Valente and Vassilicos $[27]$ as the ratio in their case becomes constant at $x / x_{\text {peak }} \approx 0.8$, which indicates that the turn to isotropy of the FSG-generated turbulence is faster. To evaluate the consistency, the ratio of $-\mathcal{A}_{\text {iso }} / \epsilon_{\text {iso }}$ for the Honda and Lille 

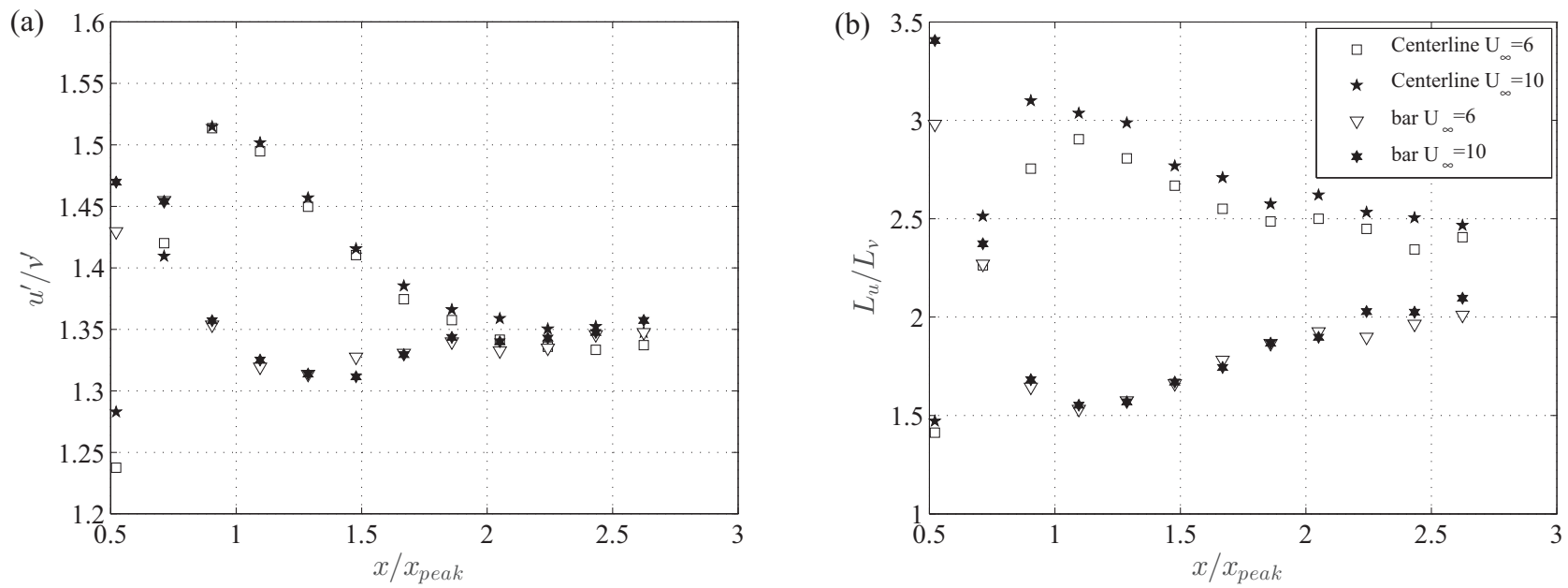

FIG. 18. Large scale isotropy criteria of (a) $u^{\prime} / v^{\prime}$, and (b) $L_{u} / L_{v}$ from the $3 \times 3$ experiment along the centerline and along the streamwise direction behind the horizontal bar of the grid.

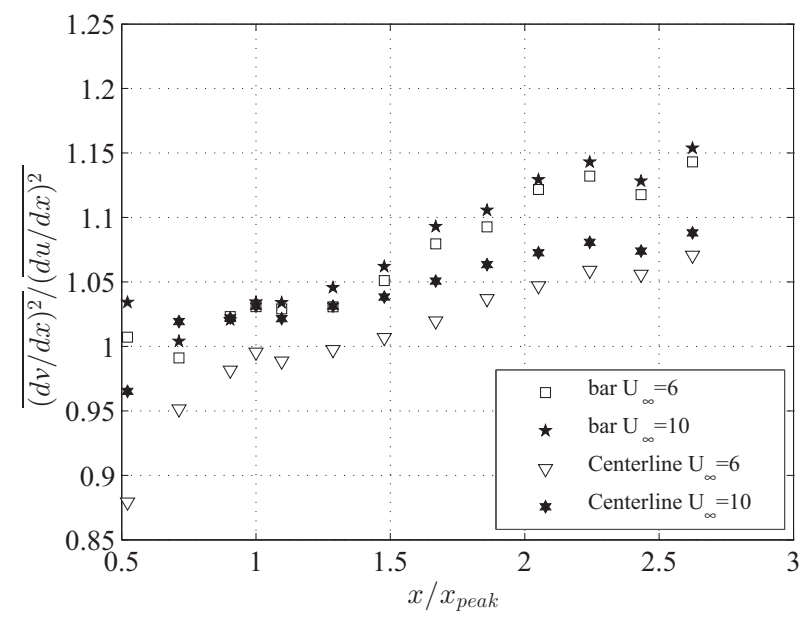

FIG. 19. The small scale isotropy $\overline{(d v / d x)^{2}} / \overline{(d u / d x)^{2}}$ from the $3 \times 3$ experiments along the centerline and the streamwise direction behind the horizontal grid bar.

measurements along the centerline are also given in figure 20 (b). It is observed that this ratio evolves in the same way, and stays close to 1 in the decay region. Following these discussions, we might argue that, although the flow is not strictly isotropic, the isotropy assumption can be used to look at the evolution of the dissipation rate as the energy contributions from these terms remain proportional. This has to exclude the region $x / x_{\text {peak }}<1.1$, which is roughly the production region of the flow.

\section{E. Non-equilibrium energy dissipation scaling}

In section III C, it has been shown that the values of $L_{u}$ and $\lambda$ increases along the streamwise direction except for a regional decrease between $x / x_{\text {peak }}=1$ to 1.5 along the centerlines. To further study the dissipation scaling, the ratio of $L_{u} / \lambda$ along the centerline and along the streamwise direction with a $y$ offset are now given in figure 21 . 

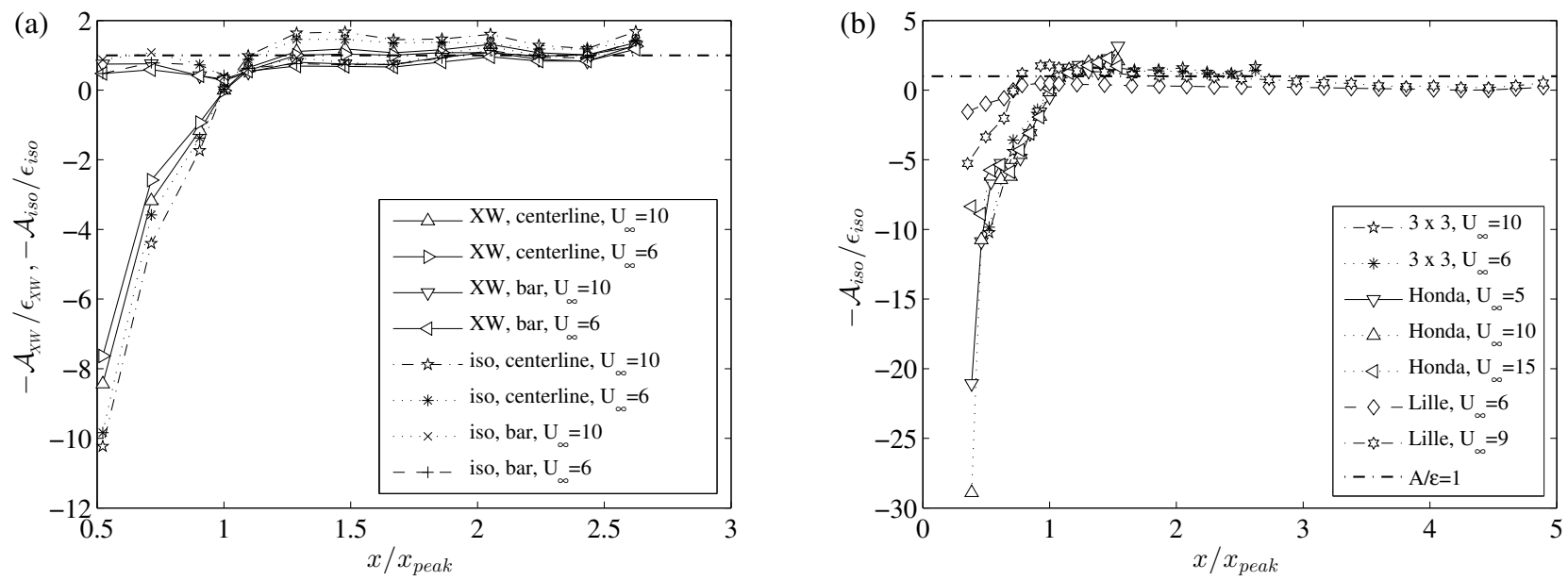

FIG. 20. Ratios of (a) $-\mathcal{A}_{X W} / \epsilon_{X W}$ and $-\mathcal{A}_{\text {iso }} / \epsilon_{\text {iso }}$ at different locations using the cross-wire measurements from the $3 \times 3$ experiments, and (b) $-\mathcal{A}_{\text {iso }} / \epsilon_{\text {iso }}$ from all three experiments using the isotropy assumption. The dashed line marks ratio of 1.
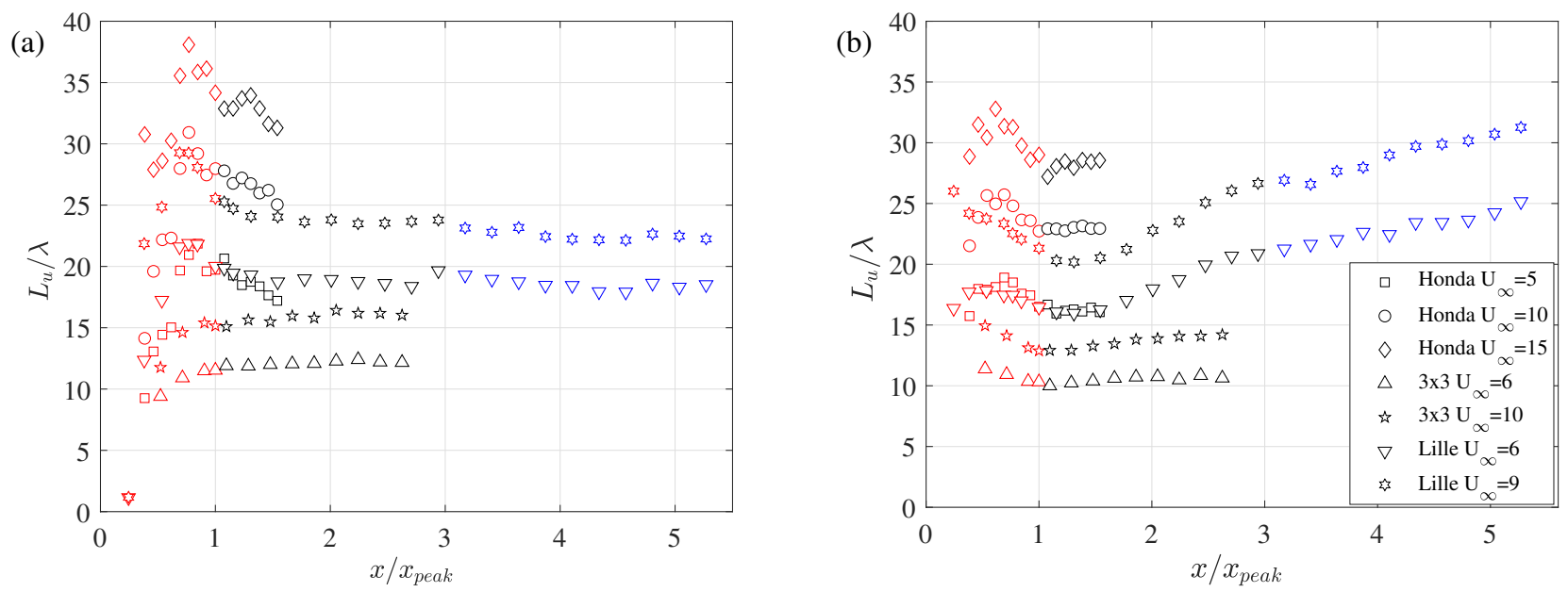

FIG. 21. Streamwise profiles of $L_{u} / \lambda$ for different inlet velocities (a) along the centerline, and (b) along the streamwise direction for the bar330 data set from Honda experiments and the bar data set from other experiments. Red and black colors mark the production and decay region, respectively. Blue marks the region in Lille experiments where $x / x_{\text {peak }}>3$.

Along the centerline, the ratio $L_{u} / \lambda$ stays roughly constant in the decay region, although results from the Honda experiments seem to decrease slightly between $x / x_{\text {peak }}=1$ to 1.5 in figure 21 (a). The variations of $L_{u} / \lambda$ from the Honda experiment at different freestream velocities are equivalent to $\pm 5 \%, \pm 3 \%$, and $\pm 2 \%$ (between 20.6 to 17.2 , 27.8 to 25.0 , and 34.0 to 31.3 ), respectively. Referring to section II D, the uncertainties of $L_{u}$ and $\lambda$ are estimated to be approximately $\pm 5 \%$ and $\pm 4 \%$, respectively, for the Honda experiment. Therefore, the decrease observed in figure 21 (a) is deemed insignificant. In the region of $x / x_{\text {peak }}=1.5$ to 3 , the constancy of $L_{u} / \lambda$ is in agreement with the non-equilibrium scalings already seen in fractal-generated turbulence $[17,18,29]$ and other turbulent flows $[30,34-36,58,59]$. Further discussions regarding the spectrum are reported in appendix B.

The blue data points in figure 21 mark the region where $R e_{\lambda}$ stays roughly constant from the Lille experiment, as shown in figure 22 (a) and (b), and therefore should not be interpreted as evidence for the non-equilibrium scaling relation. With the $y$ offset, as shown in figure $21(\mathrm{~b})$, the value of $L_{u} / \lambda$ seems roughly constant in the decay region 

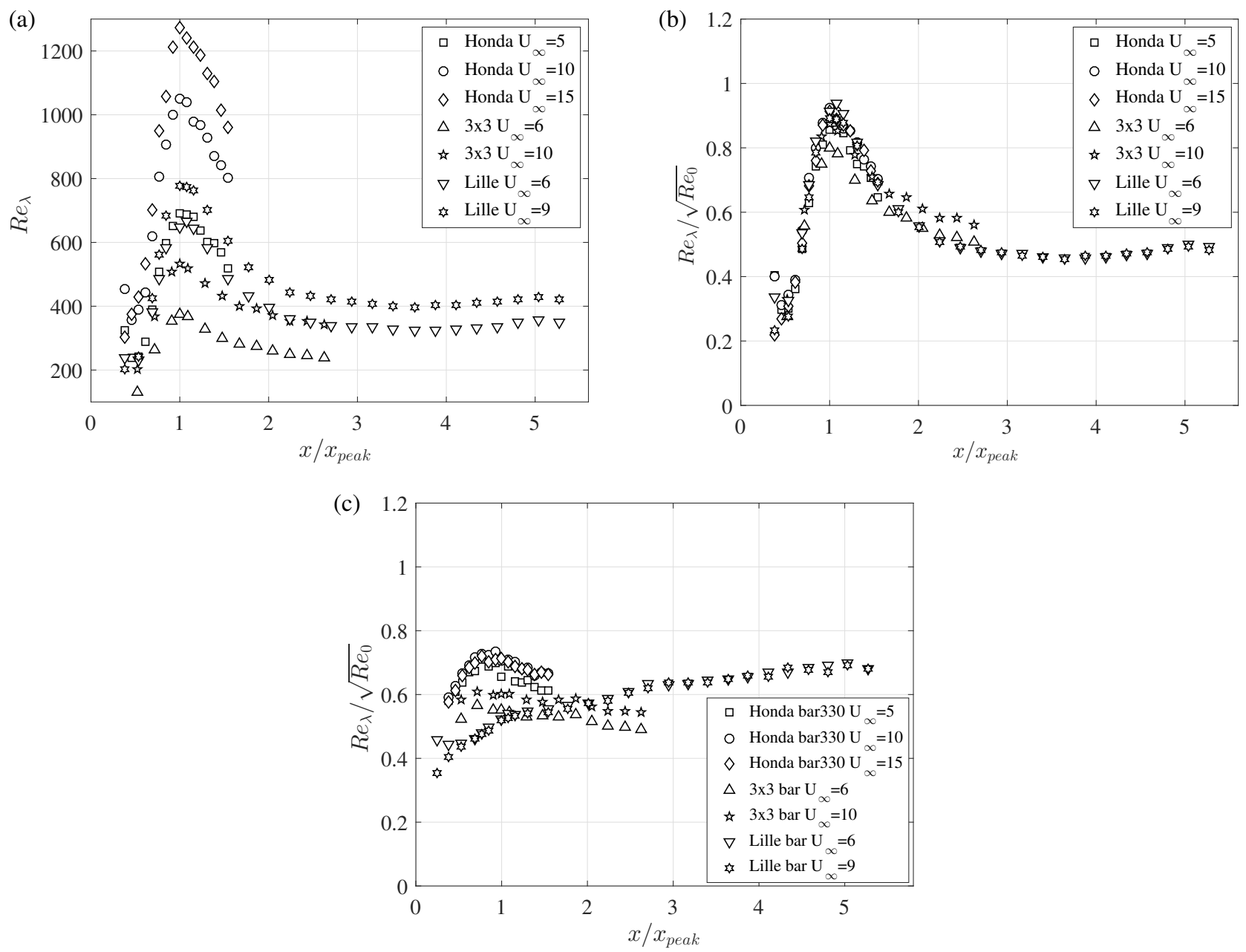

FIG. 22. Streamwise profiles of $R e_{\lambda}$ (a) along the centerline from different experiments, (b) normalized by the inlet Reynolds number $R e_{0}=U_{\infty} L_{0} / \nu$ along the centerline, and (c) normalized along the streamwise direction with $y$ offset, i.e. behind the center of the horizontal bar in $3 \times 3$ and Lille experiments (denoted by bar), and at $y=330 \mathrm{~mm}, z=0 \mathrm{~mm}$ in the Honda Experiment (denoted by bar330).

for data from Honda and $3 \times 3$ experiments, but increases monotonically in the Lille experiment. The reason for such observations can be partly explained by the growing $R e_{\lambda}$ as shown in figure 22 (c), which might be caused by the growing boundary layer.

The profiles of Taylor Reynolds number $R e_{\lambda}=u^{\prime} \lambda / \nu$ from different experiments with different $U_{\infty}$ are given in figure 22 (a). The values of $R e_{\lambda}$ increase with inlet Reynolds number $R e_{0}=U_{\infty} L_{0 z} / \nu$, and figure 22 (b) shows the collapse of $R e_{\lambda} / \sqrt{R e_{0}}$ for all the experiments along the centerline. Note that the value of $R e_{\lambda} / \sqrt{R e_{0}}$ stays roughly constant in the region of $x / x_{\text {peak }}>3$, which corresponds to the blue data points in figure 21 (a). These data points are therefore not to be interpreted as indications of the non-equilibrium dissipation scaling law.

Along the streamwise direction with $y$ offset, the normalization $R e_{\lambda} / \sqrt{R e_{0}}$ does not collapse the data as shown in figure 22 (c). It suggests that the streamwise evolution of $R e_{\lambda} / \sqrt{R e_{0}}$ varies differently along the streamwise direction off the centerline of the grid. The values of $R e_{\lambda} / \sqrt{R e_{0}}$ do not vary as much as along the centerline, and the data from the Lille experiment grow monotonically.

For turbulence generated by various grids, the Reynolds number $R e_{\lambda}$ usually decreases along the streamwise direction $x$ in the decay region [60]. The constant or increasing Reynolds numbers $R e_{\lambda}$ observed in these experiments 

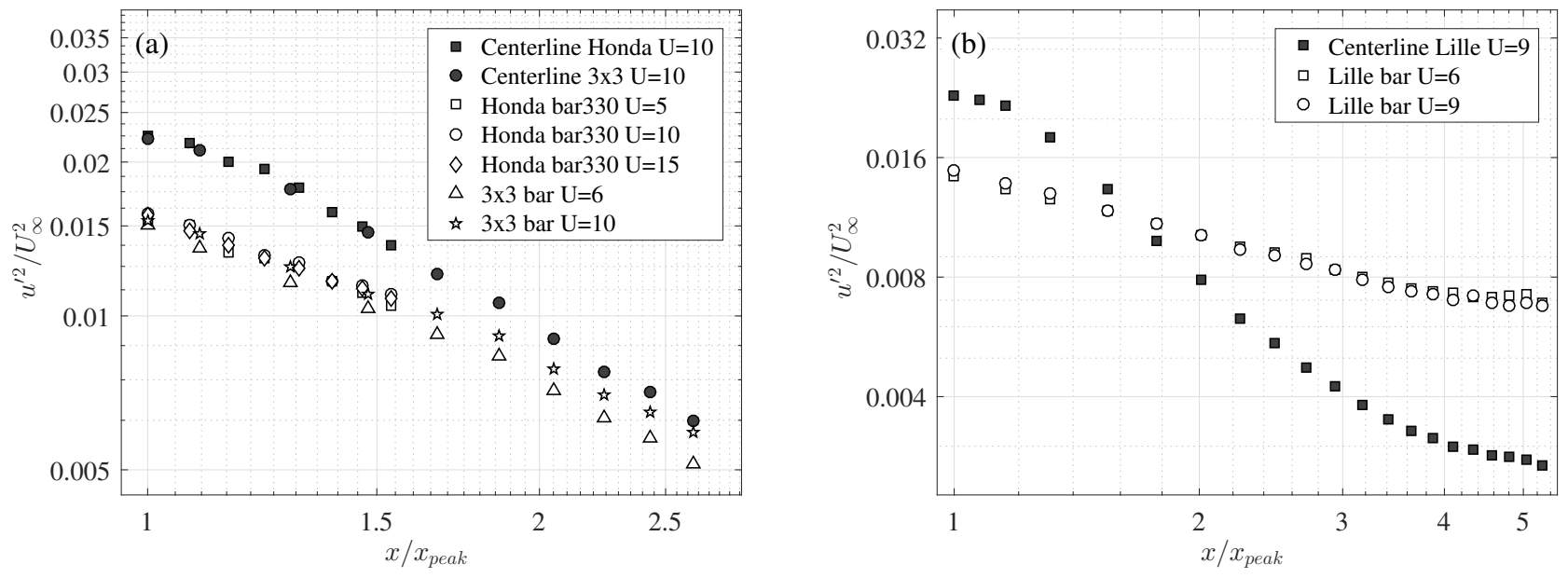

FIG. 23. Profiles of $u^{\prime 2} / U_{\infty}^{2}$ against $x / x_{\text {peak }}$ from (a) Honda and $3 \times 3$ experiments, and (b) Lille experiments, plotted in log scale.

suggest that the decreasing rate of $u^{\prime}$ is similar or smaller than the growth rate of $\lambda$. From figure 13, it can be observed that in the decay region $x / x_{\text {peak }}>2$, the growth rate of $\lambda$ is the same as they collapse in the figure. Therefore, the main reason for the differences in the evolution of $R e_{\lambda}$ perhaps lies in the different decay rates of the turbulence intensity $u^{\prime}$.

This might make sense as the growing boundary layer would increase the turbulence intensity level of the flow. In the Lille experiment, the thickness of the wall boundary layer eventually became larger than the measurement height behind the horizontal bar, implying that the decay of $u^{\prime}$ behind the bar would be much slower than that along the centerline, which is demonstrated in figure 23.

In figure 23 (a), the decay rates of the data along the centerline and those with $y$ offset appear to be similar, and their values become closer with increasing $x / x_{\text {peak }}$ distance in the Honda and $3 \times 3$ experiments. These two cases are free from any wall effects. Figure 23 (b) shows the data from the Lille experiments. It can be observed that the decay rate of the data behind the bar is much smaller than that of the centerline data, and the decay rate is reduced along the centerline from approximately $x / x_{\text {peak }}>3$. These observations support the scenario that the decay of turbulence intensity is retarded by the growing boundary layer, and it explains the slight increase of $R e_{\lambda}$ behind the horizontal bar at the end of the measurement domain such that the decay rate of $u^{\prime}$ is smaller than the growth rate of $\lambda$, as discussed regarding figure 22 (c).

Going back to the ratio of turbulence length scales, the non-equilibrium dissipation scaling relation have shown that $L_{u} / \lambda \approx$ const while $R e_{\lambda}$ decreases [27, 29,37]. The results from the current measurements along the centerline are given in figure 24 (a). The best constancy of $L_{u} / \lambda$ is observed in the decay region of $3 \times 3$ and Lille experiments, while the value of $L_{u} / \lambda$ increases with larger $R e_{0}$.

The ratio of $L_{u} / \lambda$ from the Honda experiments in the decay region seems to have a weak dependence on $R e_{\lambda}$, as observed in figure 24 (a). Referring to section II D, the uncertainties of $L_{u}$ and $\lambda$ are estimated to be approximately $\pm 5 \%$ and $\pm 4 \%$, respectively, for the Honda experiment. Therefore, the variations of $L_{u} / \lambda$ equivalent to $\pm 5 \%, \pm 3 \%$, and $\pm 2 \%$ (same as those from figure 21 a) are deemed insignificant. The data marked in blue come from the region of $x / x_{\text {peak }}>3$, where $R e_{\lambda} \approx$ const and consequently $L / \lambda \approx$ const, as shown in figure 24 (a).

Along the streamwise direction for the bar and bar330 data cases in figure 24 (b), the value of $L_{u} / \lambda$ from Honda and $3 \times 3$ experiments seems to be constant at different $R e_{\lambda}$, and increases with increasing $R e_{0}$. The data from Lille experiments follows $L_{u} / \lambda \propto R e_{\lambda}$, corresponding to the monotonic growth of $L_{u} / \lambda$ as shown in figure 21 (b). This part of data is not further interpreted as the region is expected to be affected by the boundary layer in the Lille wind tunnel.

Now if we define the dissipation coefficient as $C_{\epsilon}=\epsilon_{i s o} L_{u} / u^{\prime 3}$, and assume $\lambda^{2}=\nu u^{\prime 2} / \epsilon_{i s o}$, it implies that $L_{u} / \lambda \sim$ $C_{\epsilon} R e_{\lambda}$. For regions with $L_{u} / \lambda \approx$ const, it follows that $C_{\epsilon} \propto R e_{\lambda}^{-1}$. Normalized results along the centerline and along 

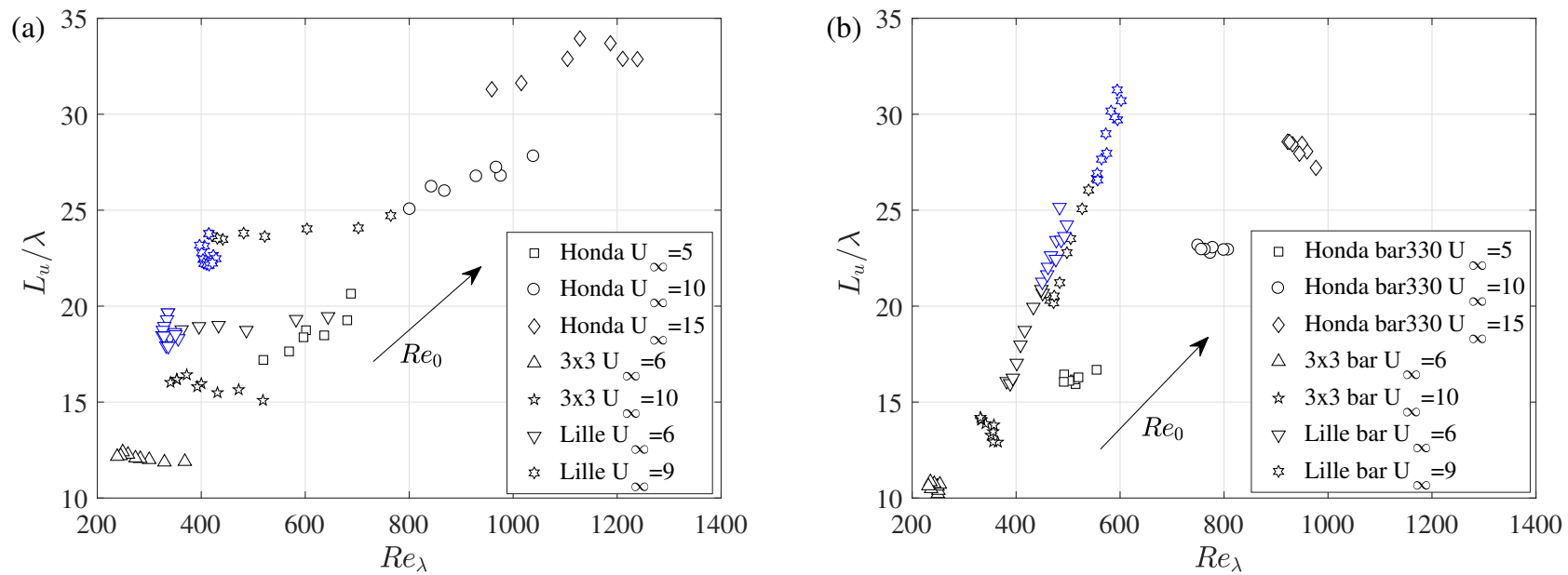

FIG. 24. Profiles of $L_{u} / \lambda$ as a function of $\operatorname{Re}_{\lambda}$ (a) along the centerline, and (b) along the streamwise direction with $y$ offset. Blue marks the region in Lille experiments where $x / x_{\text {peak }}>3$.
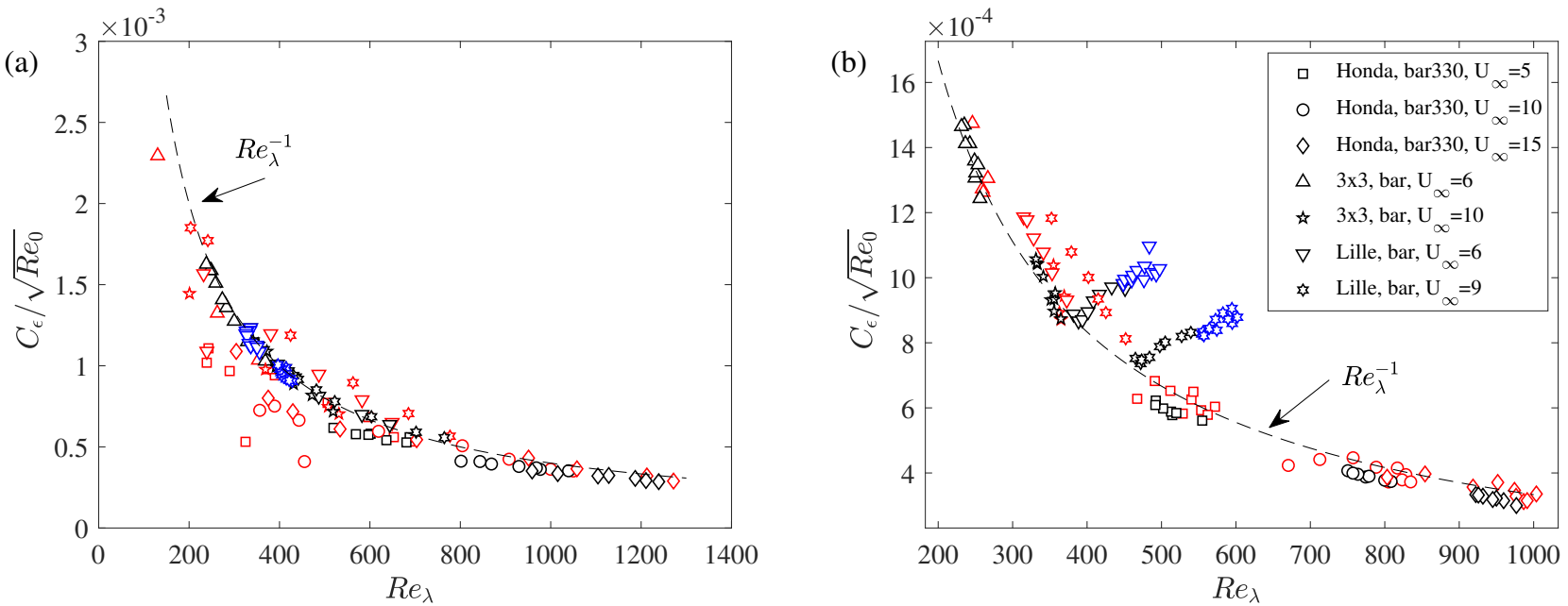

FIG. 25. Reynolds number dependence of $C_{\epsilon} / \sqrt{R e_{0}}$ along the streamwise direction on the centerline (a) and with $y$ offset (b). Red and black colors mark the production and decay region, respectively. Blue marks the region in Lille experiments where $x / x_{\text {peak }}>3$.

the streamwise direction off the centerline are given in figure 25. In both cases, $C_{\epsilon}$ decreases with increasing Reynolds number $R e_{\lambda}$, and that the values collapse with the non-equilibrium scaling such that $C_{\epsilon} \propto R e_{0}^{1 / 2} / R e_{\lambda}$, as the data collapse in figure 25 (a) for the decay region (black and blue). Figure 25 (b) gives the results off the centerline, and a clear deviation from the scaling relation can be observed in the Lille data, where the local Reynolds number $R e_{\lambda}$ slightly increases (blue symbols) as discussed regarding figure 22.

Finally, the dissipation coefficient $C_{\epsilon}$ is plotted as a function of $x / x_{\text {peak }}$ in figure 26. Figure 26 (a) has been shown in previous works [e.g. 26, 29], demonstrating the non-equilibrium scaling. However, the data from Lille experiments at $x / x_{\text {peak }}>3$ (blue symbols) should be interpreted with caution as $R e_{\lambda} \approx$ const was observed in this region, so this constancy of $C_{\epsilon}$ may not be depicted as the non-equilibrium scaling relation. Nevertheless, the values of $C_{\epsilon}$ collapse well elsewhere. Figure 26 (b) shows the results along the streamwise direction for the bar and bar330 cases. Note that 

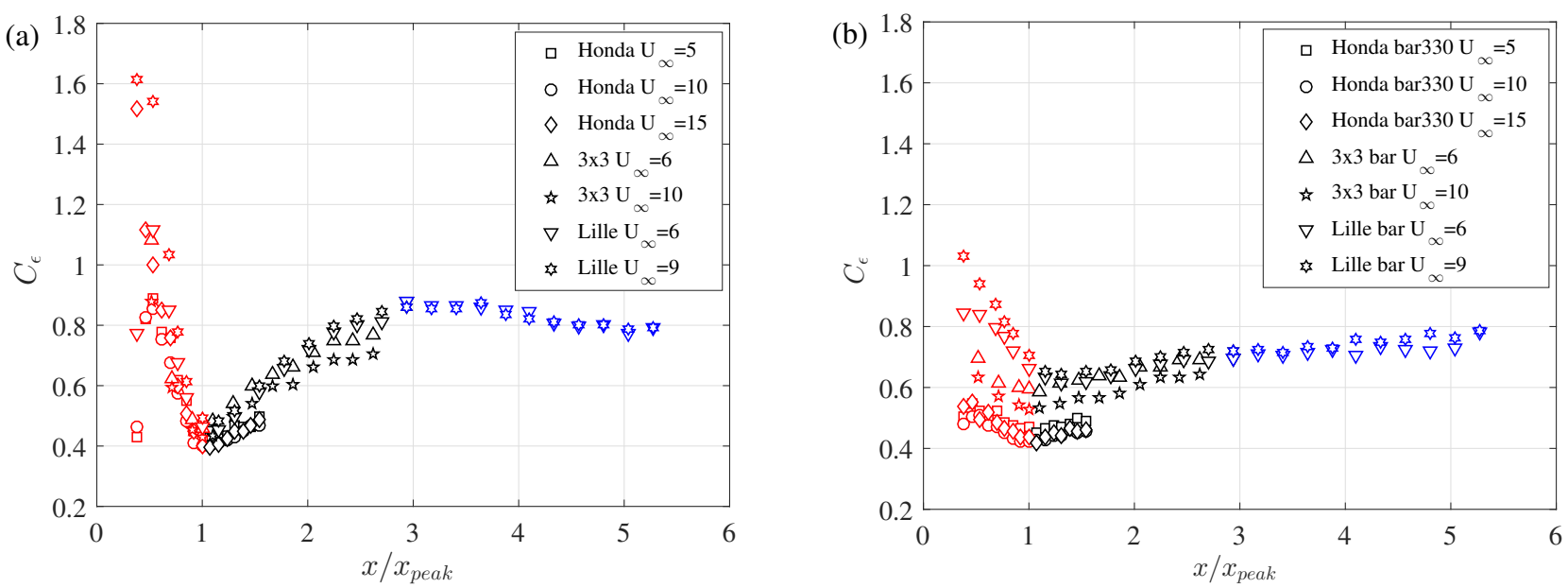

FIG. 26. Dissipation coefficient $C_{\epsilon}$ as a function of $x / x_{\text {peak }}$ (a) along the centerline, and (b) along the streamwise direction with $y$ offset. Red and black colors mark the production and decay region, respectively. Blue marks the region in Lille experiments where $x / x_{\text {peak }}>3$.

the relative measurement location in Honda experiments (bar330) and the other two is not the same, so the grouped collapse is not surprising.

\section{CONCLUSIONS AND FUTURE WORK}

In this study, a variation of the space-filling fractal square grid (FSG) was designed, referred to as the rectangular fractal grid (RFG), and tested in three different facilities to document the turbulence field.

Some technical issues regarding the hot-wire signal processing were discussed first. Two types of spectrum models were implemented to correct the large scale and small scale ranges of the spectra, respectively. The wake interaction length scale was examined, which failed to collapse the FSG and RFG data using the previous definition of $x_{*}^{\text {peak }}$. The main reason for the discrepancy is in the spreading rates of the wakes generated by grid bars, which vary for different shapes of the fractal grid. The effect can be incorporated by a scale factor $\phi=f\left(L_{0 z} / L_{0 y}\right)=0.294$ for the current grid geometry. This is critical to produce turbulent flows in laboratory with desired downstream variations.

The integral length scale $L_{u}$ and Taylor microscale $\lambda$ both showed a decreasing pattern in the beginning of the decay region, which was observed for the first time in grid-generated decaying turbulence. From comparisons with the streamwise profiles away from the centerline, it seems that the dynamic interactions of the two vortex systems from the grid bars are causing this rather unusual behavior.

In the region where decreasing length scales are observed, the flow was shown to be more inhomogeneous, even though the homogeneity for the rest of the flow is similar to those of FSG-generated turbulence. The anisotropy level in both large and small scales was shown to be greater than that for FSG generated turbulence.

To study the dissipation scaling, it was argued that the ratio of $\mathcal{A} / \epsilon$ with and without the isotropy assumption is similar, and therefore the isotropy (or constant anisotropy) assumption can be used to study the turbulence characteristics during decay. The value of $C_{\epsilon}$ in the decay region was shown to follow $C_{\epsilon} \sim R e_{0}^{1 / 2} / R e_{\lambda}$, as stated by the non-equilibrium scaling relation, regardless of the slightly poorer homogeneity and isotropy.

In general, the RFG-generated turbulence has revealed interesting features in several turbulence properties, and further work to examine the interaction between the wakes might be useful in several ways. First of all, by varying the ratio of the two lengths of the largest grid bars, the spreading rate of the wakes can be revisited to quantify the scaling factor $\phi=f\left(L_{0 z} / L_{0 y}\right)$. This scaling factor can be used to generalize the definition of the wake interaction length scale to prescribe the turbulence peak location $x_{\text {peak }}$, which can be of practical use in various wind tunnel applications. Further measurements of the flow structures and terms in the turbulent kinetic energy equation in the beginning of 
the decay region may help elucidate the interaction mechanism between the two vortex systems generated by the two distinct large length scales of the grid bars, and ultimately contribute to the general understanding of grid-generated turbulence.

\section{ACKNOWLEDGMENT}

The authors gratefully acknowledge the support from Marie Curie FP7 through the MULTISOLVE project, grant number 317269. JCV also acknowledges support from ERC Advance Grant 320560. The Lille experimental work was carried out within the framework of the CNRS Research Foundation on Ground Transport and Mobility, in articulation with the ELSAT2020 project supported by the European Community, the French Ministry of Higher Education and Research, the Hauts de France Regional Council.

[1] L. F. G. Simmons and C. Salter, Experimental investigation and analysis of the velocity variations in turbulent flow, Proc. R. Soc. Lon. Ser.-A 145, 212 (1934).

[2] G. I. Taylor, Statistical theory of turbulence, Proc. R. Soc. Lon. Ser.-A 151, 421 (1935).

[3] G. K. Batchelor, The theory of homogeneous turbulence (Cambridge university press, 1953).

[4] H. L. Grant and I. C. T. Nisbet, The inhomogeneity of grid turbulence, J. Fluid Mech. 2, 263 (1957).

[5] G. Comte-Bellot and S. Corrsin, The use of a contraction to improve the isotropy of grid-generated turbulence, J. Fluid Mech. 25, 657 (1966), power law decay.

[6] G. Comte-Bellot and S. Corrsin, Simple eulerian time correlation of full-and narrow-band velocity signals in grid-generated, 'isotropic' turbulence, J. Fluid Mech. 48, 273 (1971).

[7] M. Gad-El-Hak and S. Corrsin, Measurements of the nearly isotropic turbulence behind a uniform jet grid, J. Fluid Mech. 62, 115 (1974).

[8] L. Mydlarski and Z. Warhaft, On the onset of high-reynolds-number grid-generated wind tunnel turbulence, J. Fluid Mech. 320, 331 (1996).

[9] J. I. Cardesa, T. B. Nickels, and J. R. Dawson, 2D PIV measurements in the near field of grid turbulence using stitched fields from multiple cameras, Exp. Fluids 52, 1611 (2012).

[10] R. E. Seoud and J. C. Vassilicos, Dissipation and decay of fractal-generated turbulence, Phy. Fluids 19, 1 (2007).

[11] J. C. Isaza, R. Salazar, and Z. Warhaft, On grid-generated turbulence in the near- and far field regions, J. Fluid Mech. 753, 402 (2014).

[12] P. C. Valente and J. C. Vassilicos, The non-equilibrium region of grid-generated decaying turbulence, J. Fluid Mech. 744, 5 (2014).

[13] J. C. Vassilicos, Intermittency in turbulent flows (Cambridge University Press, 2001).

[14] B. Mazzi, F. Okkels, and J. C. Vassilicos, A shell-model approach to fractal-induced turbulence, Eur. Phys. J. B 28, 243 (2002).

[15] B. Mazzi and J. C. Vassilicos, Fractal-generated turbulence, J. Fluid Mech. 502, 65 (2004).

[16] D. Hurst and J. C. Vassilicos, Scalings and decay of fractal-generated turbulence, Phys. Fluids 19, 035103 (2007).

[17] N. Mazellier and J. C. Vassilicos, Turbulence without richardson-kolmogorov cascade, Phys. Fluids 22, 075101 (2010).

[18] R. Gomes-Fernandes, B. Ganapathisubramani, and J. C. Vassilicos, Particle image velocimetry study of fractal-generated turbulence, J. Fluid Mech. 711, 306 (2012).

[19] A. A. Townsend, The structure of turbulent shear flow (Cambridge university press, 1980).

[20] C. R. Symes and L. E. Fink, Effects of external turbulence upon the flow past cylinders, in Structure and mechanisms of turbulence I (Springer, 1977) pp. 86-102.

[21] P. W. Bearman and D. M. Trueman, Investigation of flow around rectangular cylinders, Aeronaut. Quart. 23, 229 (1972).

[22] Y. Nakamura and Y. Tomonari, The effect of turbulence on the drags of rectangular prisms, T. JPN Soc. Aeronaut. S. 19, $82(1976)$.

[23] S. B. Pope, Turbulent flows (IOP Publishing, 2001).

[24] K. R. Sreenivasan, On the scaling of the turbulence energy dissipation rate, Phys. Fluids 27, 1048 (1984).

[25] J. L. Lumley, Some comments on turbulence, Phys. Fluids A-Fluid 4, 203 (1992).

[26] J. C. Vassilicos, Dissipation in turbulent flows, Annu. Rev. Fluid. Mech. 47, 95 (2015).

[27] P. C. Valente and J. C. Vassilicos, The decay of turbulence generated by a class of multiscale grids, J. Fluid Mech. 687, $300(2011)$. 
[28] D. Stefano, B. Z. Isaac, A. Tommaso, J. A. Ronald, and P. P. Kathy, Piv measurements of anisotropy and inhomogeneity in decaying fractal generated turbulence, Fluid Dyn. Res. 45, 061401 (2013).

[29] R. J. Hearst and P. Lavoie, Decay of turbulence generated by a square-fractal-element grid, J. Fluid Mech. 741, 567 (2014).

[30] S. Goto and J. C. Vassilicos, Energy dissipation and flux laws for unsteady turbulence, Phys. Lett. A 379, 1144 (2015).

[31] S. Goto and J. C. Vassilicos, Local equilibrium hypothesis and taylor's dissipation law, Fluid Dyn. Res. 48, 021402 (2016).

[32] M. Obligado, T. Dairay, and J. C. Vassilicos, Nonequilibrium scalings of turbulent wakes, Phys. Rev. Fluids 1, 044409 (2016).

[33] K. Nagata, T. Saiki, Y. Sakai, Y. Ito, and K. Iwano, Effects of grid geometry on non-equilibrium dissipation in grid turbulence, Phys. Fluids 29, 015102 (2017).

[34] J. Nedić, S. Tavoularis, and I. Marusic, Dissipation scaling in constant-pressure turbulent boundary layers, Phys. Rev. Fluids 2, 032601(R) (2017).

[35] K. Takamure, Y. Sakai, Y. Ito, K. Iwano, and T. Hayase, Dissipation scaling in the transition region of turbulent mixing layer, Int. J. Heat Fluid Fl. 75, 77 (2019).

[36] G. Cafiero and J. C. Vassilicos, Non-equilibrium turbulence scalings and self-similarity in turbulent planar jets, Proceedings of the Royal Society A: Mathematical, Physical and Engineering Sciences 475, 20190038 (2019).

[37] P. C. Valente and J. C. Vassilicos, Universal dissipation scaling for nonequilibrium turbulence, Phys. Rev. Lett. 108, 214503 (2012).

[38] S. Zheng, Turbulence genrated by inhomogeneous and multiscale grids, Ph.D. thesis, Imperial College London (2017).

[39] R. M. Lueptow, K. S. Breuer, and J. H. Haritonidis, Computer-aided calibration of x-probes using a look-up table, Exp. Fluids 6, 115 (1988).

[40] K. Bremhorst, Effect of fluid temperature on hot-wire anemometers and an improved method of temperature compensation and linearisation without use of small signal sensitivities, J. Phys. E Sci. Instrum. 18, 44 (1985).

[41] P. L. O'Neill, D. Nicolaides, D. Honnery, and J. Soria, Autocorrelation functions and the determination of integral length with reference to experimental and numerical data, in Proc. 15th Australasian Fluid Mechanics Conference, Sydney, Australia (2004).

[42] T. von Kármán, The fundamentals of the statistical theory of turbulence, J. Aeronaut. Sci. 4, 131 (1937).

[43] D. K. Wilson, A New Model for Turbulence Spectra and Correlations Based on Meijer's G-Functions, Technical Note ARL-TN-104 (Army Research Laboratory, 1998).

[44] G. S. Seyed and V. V. Srinivas, Hot-wire anemometry behaviour at very high frequencies, Meas. Sci. Technol. 7, 1297 (1996).

[45] R. A. Antonia, On estimating mean and instantaneous turbulent energy dissipation rates with hot wires, Exp. Therm. Fluid Sci. 27, 151 (2003).

[46] T. Sanada, Comment on the dissipation-range spectrum in turbulent flows, Phys. Fluids A-Fluid 4, 1086 (1992).

[47] R. A. Antonia, H. S. Shafi, and Y. Zhu, A note on the vorticity spectrum, Phys. Fluids 8, 2196 (1996).

[48] B. R. Pearson, Experiments on small-scale turbulence, Ph.D. thesis, University of Newcastle (1999).

[49] T. Zhou, R. Antonia, and L. Chua, Performance of a probe for measuring turbulent energy and temperature dissipation rates, Exp. Fluids 33, 334 (2002).

[50] J. Mi, M. Xu, and C. Du, Digital filter for hot-wire measurements of small-scale turbulence properties, Meas. Sci. Technol. 22, 125401 (2011).

[51] I. Wygnanski, F. Champagne, and B. Marasli, On the large-scale structures in two-dimensional, small-deficit, turbulent wakes, J. Fluid Mech. 168, 31 (1986).

[52] W. K. George, The self-preservation of turbulent flows and its relation to initial conditions and coherent structures, Adv. turb. , 39 (1989).

[53] K. Nagata, Y. Sakai, T. Inaba, H. Suzuki, O. Terashima, and H. Suzuki, Turbulence structure and turbulence kinetic energy transport in multiscale/fractal-generated turbulence, Phys. Fluids 25, 065102 (2013).

[54] Y. Zhou, K. Nagata, Y. Sakai, H. Suzuki, Y. Ito, O. Terashima, and T. Hayase, Relevance of turbulence behind the single square grid to turbulence generated by regular- and multiscale-grids, Phys. Fluids 26, 075105 (2014).

[55] S. Laizet, J. Nedic, and J. C. Vassilicos, The spatial origin of $-5 / 3$ spectra in grid-generated turbulence, Phys. Fluids 27, 065115 (2015).

[56] S. Corrsin, Turbulence: experimental methods, Handbuch der Physik 3, 524 (1963).

[57] H. S. Kang, S. Chester, and C. Meneveau, Decaying turbulence in an active-grid-generated flow and comparisons with large-eddy simulation, J. Fluid Mech. 480, 129 (2003).

[58] T. Dairay, M. Obligado, and J. C. Vassilicos, Non-equilibrium scaling laws in axisymmetric turbulent wakes, J. Fluid Mech. $\mathbf{7 8 1}, 166(2015)$.

[59] S. Goto and J. C. Vassilicos, Unsteady turbulence cascades, Phys. Rev. E 94, 053108 (2016).

[60] G. Melina, P. J. K. Bruce, and J. C. Vassilicos, Vortex shedding effects in grid-generated turbulence, Phys. Rev. Fluids 1, $044402(2016)$. 


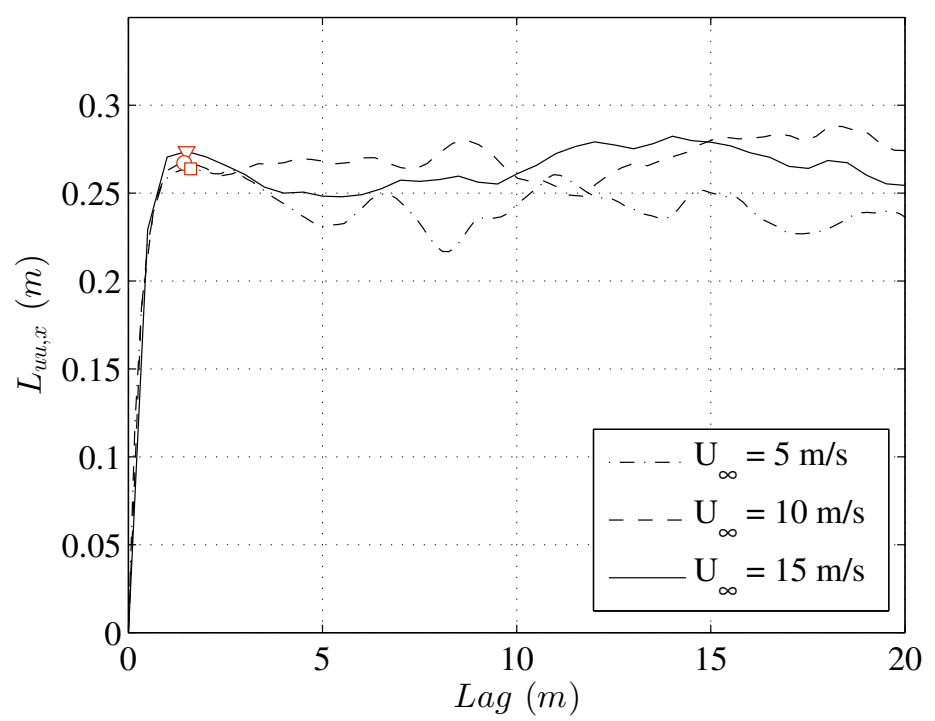

FIG. 27. Convergence of $L_{u}$ with increasing integral domain. The symbols give the results by integrating the correlation function only up to the first zero-crossing.

\section{Appendix A: Integral length scale}

In order to calculate the longitudinal integral length scales $L_{u}$, the correlation function was examined. It is noticed that the auto-correlation function drops below zero and vibrates around $R=0$ as the separation distance increases. This phenomenon raises the question of the integration domain, which affects the calculation result of $L_{u}$ significantly. [see 41] discussed several methods using either the entire available domain, or up to where $R=R_{\min }$, or up to the first zero-crossing if negative $R$ is present, and they concluded that there may not be a clear relation between the integral length scale and the integration domain. For the discussion here, the first zero-crossing point is used for comparison. Figure 27 shows the variation of $L_{u}$ as a function of the integration domain as well as the results by integrating only up to the first zero-crossing (red symbols). It is hard to argue whether the values integrated up to the first zero-crossing point are representative due to the large variations with increasing integration domain.

Another popular method to calculate $L_{u}$ is to divide the sample into smaller piecewise samples, calculate $L_{u}$ for each piecewise sample by integrating up to the first zero-crossing point, and then take an average. This method, however, is sensitive to the selection of the sample piece length, as shown in figure 28. It is clear that for pieces with $d t$ larger than $5 \mathrm{~s}$, where $d t$ is the sample time length for each piece, the results are converged. Also notice that the streamwise development of $L_{u}$ is fluctuating more than expected, and the discrepancies of the results using different piecewise length at given location is about $10 \%$. This seems to imply the ambiguity of this integration method, as the integration domain cannot be well defined. Therefore the spectrum method discussed in section IIC is used.

\section{Appendix B: Self similarity of spectra}

In the decaying homogeneous and isotropic turbulence, the classical equilibrium theory states that the spectra are expected to collapse at small scales when normalized using inner variables ( $\epsilon$ and $\eta)$, and at large scales using outer variables $\left(\overline{u^{2}}\right.$ and $\left.L_{u}\right)$. For turbulent flows with the non-equilibrium scaling such that $L_{u} / \lambda \approx$ const, it has been shown that either $L_{u}$ or $\lambda$ can be used to collapse the large-scale range and inertial range of energy spectra, corresponding to a single-length scale spectra $E_{11}(k)=u^{\prime 2} l F_{11}(k l)$ in those two ranges, where $l$ is a characteristic length scale [17, 27, 29]. 


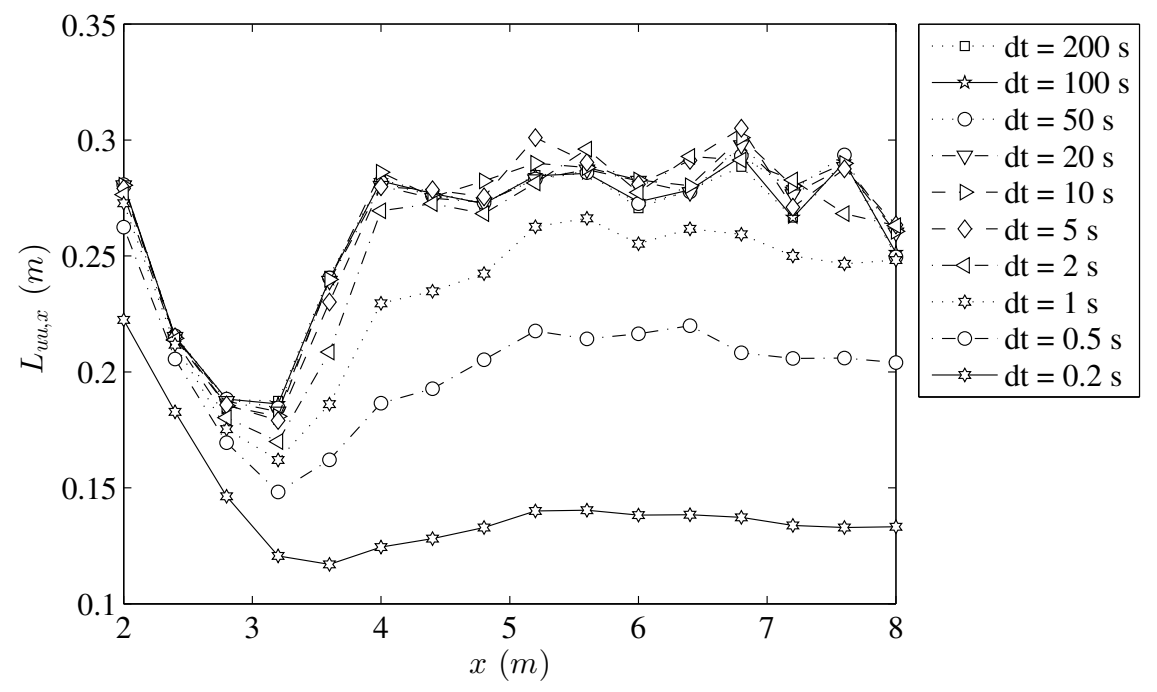

FIG. 28. Convergence of piecewise length in the calculation of $L_{u}$ at $U_{\infty}=10 \mathrm{~m} \mathrm{~s}^{-1}$.

Mazellier and Vassilicos [17] also showed a dependence of the spectra on the initial conditions, such that $E_{11}(k)=$ $u^{\prime 2} l F_{11}\left(k l, R e_{0}, *\right)$, where the initial condition is characterized by $R e_{0}$, and $*$ represents any other possible factors. To evaluate this scaling, compensated spectra at given $R e_{0}$ are plotted at different streamwise locations, as shown in figure 29 .

The spectra are compensated with the $-5 / 3$ slope, and the inertial subrange shows a clear plateau in all cases. In figure 29 (a) and (b), a weak dependence of $L_{u} / \lambda$ on Reynolds number in the Honda experiment is observed, such that the small dissipative scales are not collapsed when normalized using $u^{\prime}$ and $L_{u}$. This agrees with the previous discussion on figure 21 . In the $3 \times 3$ experiments, it has been shown that, even though $L_{u}$ and $\lambda$ decrease individually in the beginning of the decay region, the ratio of $L_{u} / \lambda$ is roughly constant. This corresponds to the well collapsed spectra in the entire range as shown in figure 29 (c) and (d). A closer examination of the spectra of the Lille data, as shown in figure 29 (e) and (f), reveals that the data are not perfectly collapsed at $x / x_{\text {peak }}<1.5$, and those after $x / x_{\text {peak }}=2.5$ collapse better. Referring back to figure 21 (a), one could indeed argue that the value of $L_{u} / \lambda$ decreases slightly immediately after $x / x_{\text {peak }}=1$, but the rest of the data falls into the non-equilibrium regime nevertheless. The collapse of the spectra at $x / x_{\text {peak }}>3$ is mainly due to the roughly constant Reynolds number $R e_{\lambda}$ as discussed in the previous section.

To examine the dependence on $R e_{0}$, and to avoid the effect of the region $1<x / x_{\text {peak }}<1.5$, selected data from $3 \times 3$ and Lille experiments at $x / x_{\text {peak }} \approx 2.45$ are plotted in figure 30 , where it can be seen that they do not collapse in the dissipation range. Note that the spread of spectra in the dissipative range is smaller for normalization using the inner variables. This also agrees with the analysis proposed by Valente and Vassilicos [27], such that for a given Reynolds number ratio $R e_{\lambda_{1}} / R e_{\lambda_{2}}$ the spread using outer variables are about 3 times larger than that using inner variables. 

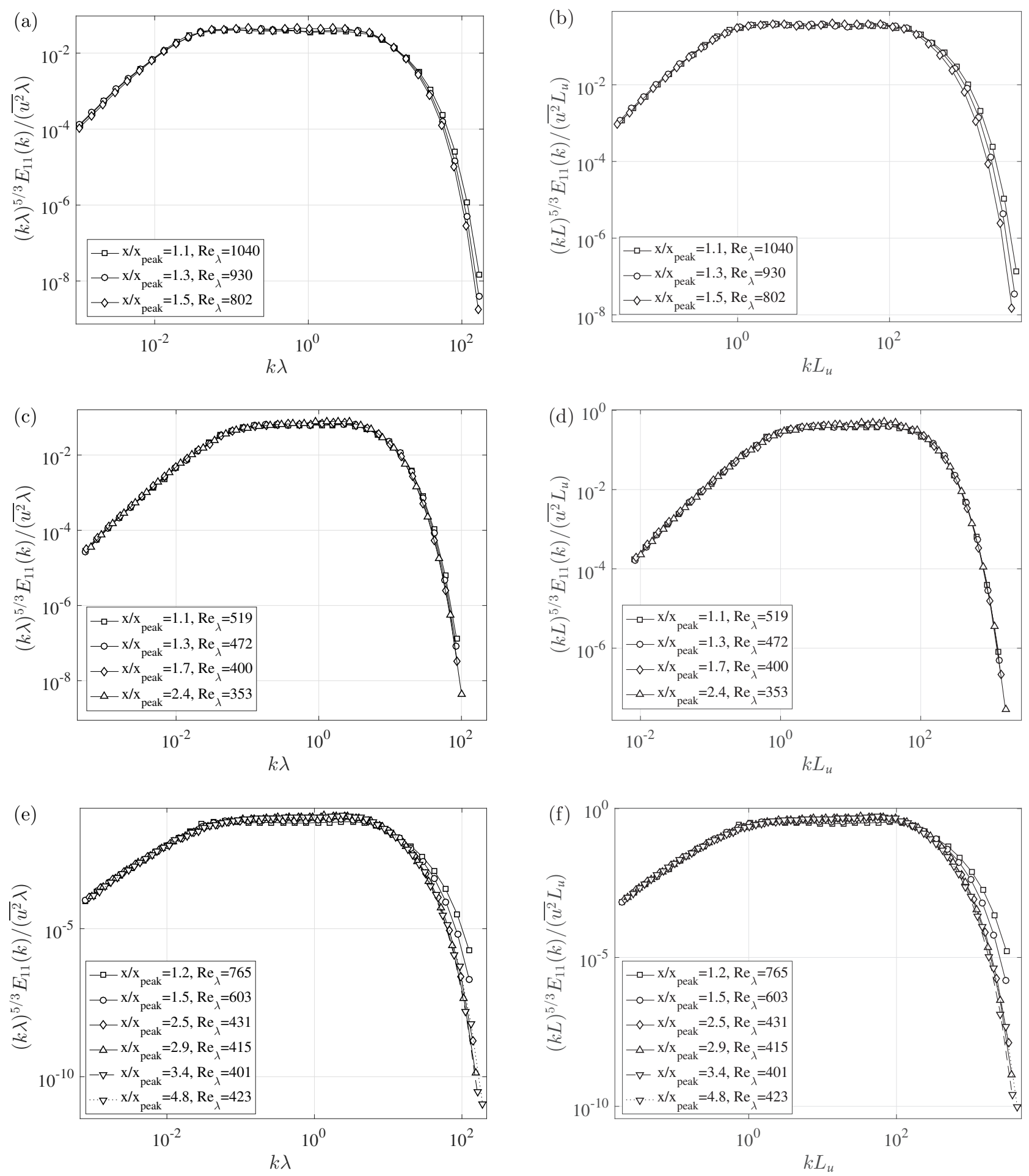

FIG. 29. Compensated one-dimensional spectra along the centerline using the inner variables (left) and outer variables (right) at different streamwise locations normalized as $x / x_{\text {peak }}$ from Honda experiments with $U_{\infty}=10 \mathrm{~m} / \mathrm{s}(\mathrm{a}, \mathrm{b}), 3 \times 3$ experiments with $U_{\infty}=10 \mathrm{~m} / \mathrm{s}(\mathrm{c}, \mathrm{d})$, and Lille experiments with $U_{\infty}=9 \mathrm{~m} / \mathrm{s}(\mathrm{e}, \mathrm{f})$. 

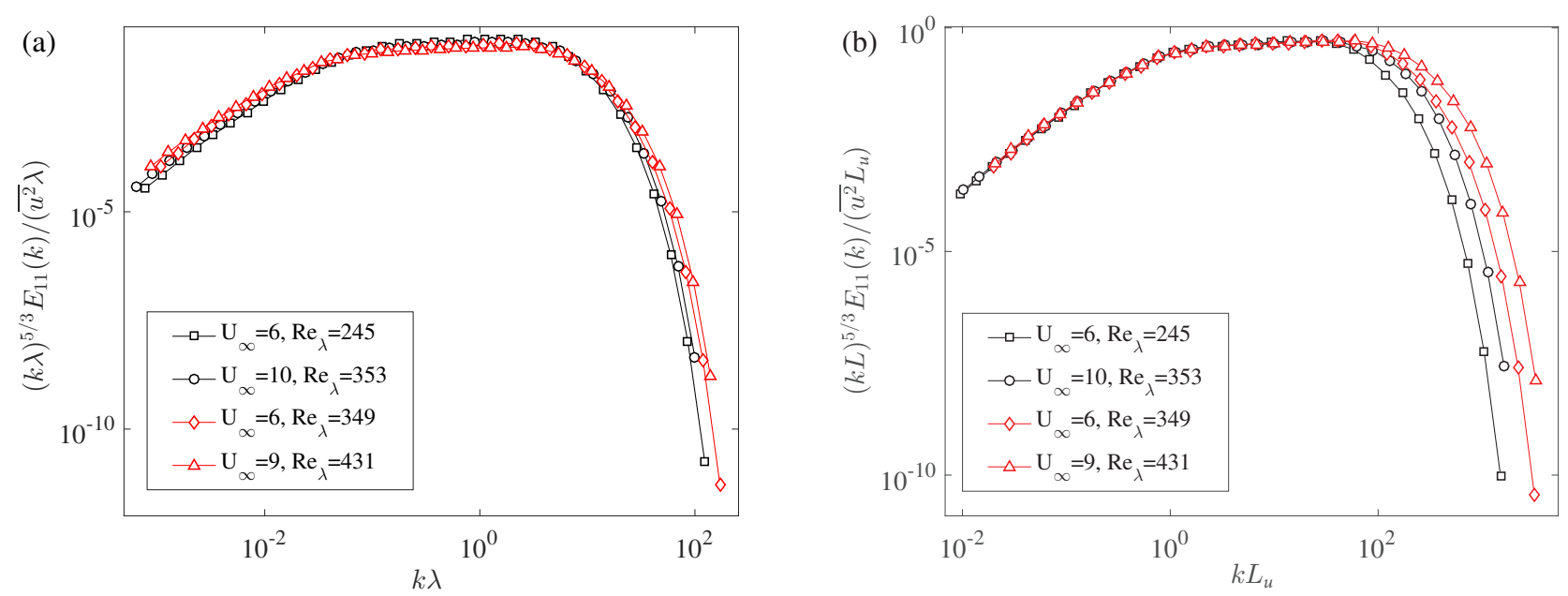

FIG. 30. Compensated one-dimensional spectra along the centerline using (a) inner variables and (b) outer variables at $x / x_{\text {peak }} \approx 2.45$ with different $R e_{0}$ from $3 \times 3$ experiments with $U_{\infty}=6 \mathrm{~m} / \mathrm{s}$ and $10 \mathrm{~m} / \mathrm{s}$, and Lille experiments with $U_{\infty}=6 \mathrm{~m} / \mathrm{s}$ and $9 \mathrm{~m} / \mathrm{s}$. 